\title{
The Carbohydrate and Caloric Content of Concomitant Medications for Children with Epilepsy on the Ketogenic Diet
}

\author{
Denis Lebel, Caroline Morin, Micheline Laberge, Nathalie Achim and \\ Lionel Carmant
}

\begin{abstract}
Background: The ketogenic diet for children with refractory epilepsy requires a strict control of the amount of ingested carbohydrates. This can be altered by medication prescribed for the epileptic syndrome or for intercurrent illnesses. The goal of this paper is to compile the carbohydrate and caloric content of commonly used medications in this population. Methods: We compiled a list of frequently used medications with the help of Canadian manufacturers and the Compendium of Pharmaceuticals and Specialties. We also tested a worst case scenario calculation based on the weight of the tablet. Results: We list the carbohydrate and caloric content of 790 medications studied. Our worst case scenario gives an over-estimate in all cases, making adjustments based on this calculation in an emergency setting safe. Conclusion: We propose this list as a tool for physicians, dietitians, nurses and pharmacists. The list can easily be adjusted, based on local practices and reviewed periodically.
\end{abstract}

\begin{abstract}
RÉSUMÉ: Le contenu en hydrates de carbone et en calories des médicaments concomitants chez les enfants épileptiques suivant la diète cétogène. Introduction: La diète cétogène chez les enfants dont l'épilepsie est résistante au traitement demande un contrôle strict de la quantité d'hydrates de carbone ingérée qui peut être influencée par la médication prescrite pour le syndrome épileptique ou pour une maladie intercurrente. Le but de cet article est de compiler le contenu en hydrates de carbone et en calories de médicaments d'usage courant dans cette population. Méthodes: Nous avons compilé une liste des médicaments d'usage courant avec l'aide de manufacturiers Canadiens et du Compendium des produits et spécialités pharmaceutiques. Nous avons également testé un calcul basé sur le poids du comprimé qui tenait compte de la pire situation possible. Résultats: Nous avons dressé une liste du contenu en hydrates de carbone et en calories de 790 médicaments. Notre calcul selon la pire situation surestime dans tous les cas, ce qui assure la sécurité de l'ajustement basé sur ce calcul en situation d'urgence. Conclusions: Nous proposons cette liste comme outil pour les médecins, les diététistes, les infirmières et les pharmaciens. La liste peut facilement être ajustée selon les pratiques locales et révisée périodiquement.
\end{abstract}

Can. J. Neurol. Sci. 2001; 28: 322-340

The ketogenic diet is a form of treatment used in children, and sometimes adults, with refractory epilepsies or with significant adverse events secondary to the anticonvulsants. ${ }^{1,2}$ The first report on the diet came in 1911 and it was supported by a second report 10 years later $^{3,4}$ but the diet became almost obsolete following the release of new medications in the 1940s. Due to the partial efficacy of phenytoin, further efforts to improve the diet were later introduced leading to better compliance and again encouraging results. ${ }^{5}$ Once again, in the '70s, the advent of new medications particularly valproic acid, lessened the interest in the diet. ${ }^{6}$ Over the past 10 years, because of its persistent efficacy in children with refractory and even more benign epilepsies, there has been increasing interest from families, clinicians and researchers to use the diet to a greater extent and to better understand it. ${ }^{6}$

Although its mechanisms of action are still not understood, we now realize that complete or even partial loss of ketosis can impair seizure control. ${ }^{7}$ Strict compliance with the diet is, therefore, of foremost importance. Some animal models show an increased seizure threshold during treatment with the diet. ${ }^{8} \mathrm{We}$ also know that ketone bodies are present in the blood and are able to cross the blood brain barrier, to serve as the principal source of energy to the brain. However, electrophysiological studies do not indicate that decreased neuronal excitability is mediated by a direct effect of ketone bodies. ${ }^{9}$ Ketosis is

\footnotetext{
From the Department of Pharmacy (DL, CM, NA) and Pediatrics (ML, LC), Hôpital Ste-Justine, University of Montreal, Montreal, QC Canada ReCeived DeCember 14, 2000. ACCePted infinalformunne 20, 2001. Reprint requests to: Lionel Carmant, Hôpital Ste-Justine, Department of Pediatrics, Division of Neurology, 3175 Côte Ste-Catherine, Montreal, QC, Canada H3T1C5
} 
established by the activation of fatty acid oxidation, secondary to the lack of carbohydrate reserves induced by the starvation period and maintained by the high lipid/carbohydrate + protein content of the diet. The constituents of the diet are calculated to provide a ratio of lipids/proteins + carbohydrates of 3/1 to 4/1.

Carbohydrates can only make up $20 \%$ of total calorie intake in a diet already limited on calorie supply, compared to more than $50 \%$ in a normal diet. The diet is usually maintained for a minimum period of two to six months and, if associated with a significant improvement, is pursued for at least two years. During this two-year period, infectious illnesses and other medical problems are common in a population of children with refractory epilepsy often with an associated mental handicap. Although syrups are usually contra-indicated due to their high carbohydrate content in the form of sucrose, maltose, sorbitol, mannitol, alcohol or starch, the "sugar free" label of drug tablets does not guarantee that ketosis will not be affected. The "sugar free" label is used primarily for diabetics and these tablets may contain sorbitol, a carbohydrate which will not affect glycemia but mightl affect ketosis in the diet. The carbohydrate content of tablets, including anticonvulsants and other concomitant drugs, can be difficult to manage for the physicians, nurses and pharmacists. Published material from the US has been reviewed but cannot be used in Canada because the drug formulations are different in the US. ${ }^{10}$ The goal of this paper is to review the carbohydrate and calorie content of the drugs most commonly used by children to help clinicians evaluate their antiketotic effects.

\section{Methods}

Between November 1998 and November 1999 we listed all drugs used on the neurology and intensive care wards that are available in liquid form, especially anticonvulsants, antibiotics, analgesics, antipyretic drugs, anti-inflammatory medications, laxatives and vitamins. We also reviewed tablet and capsule forms of anticonvulsants, antibiotics, and laxatives. Their monography was reviewed in the Compendium of Pharmaceuticals and Specialties (CPS) and each company was then contacted by mail to complete missing data about the drug's content of carbohydrates and their derivatives, as well as their calorie content. We sent a total of 894 requests for information.

On 46 drugs we tested a worst case scenario formula to evaluate the maximal carbohydrate content of a tablet or capsule. We measured the weight of 10 tablets, subtracted the amount of active material in these tablets and then divided the result by the number of tablets weighed. We considered this number to represent the maximal carbohydrate content of the pharmaceutical formulation in grams and extrapolated the energy content by multiplying the calculated number by 4 kCalories.

\section{RESUlTS}

Thanks to the collaboration of most of the pharmaceutical companies, we were able to compile the data published in Table 1. This is, we believe, a useful tool for pharmacists, nurses and physicians involved in the care of these children. The list of drugs can easily be increased based on local practices and reviewed after a few years.
The results of the worst case scenario calculations showed that we never underestimated the content of a tablet or capsule (Table 2). The tendency is actually to overestimate the carbohydrate content of the drugs. For example, a Biaxin tablet, with $250 \mathrm{mg}$ of active ingredient weighs $0,520 \mathrm{~g}$. When we substract the active ingredient, we have $270 \mathrm{mg}$ left. The worst case scenario assumes that all of this weight is made of carbohydrates. When we compare the results of the worst case scenario $(1.08 \mathrm{kCal})$ to the data obtained from the manufacturer $(0.210 \mathrm{kCal})$, we found that the worse case scenario overestimates the caloric content.

\section{Discussion}

\section{Carbohydrates and loss of ketosis.}

The ingestion of an excessive amount of carbohydrate in the diet stimulates the release of insulin, which stops the release of free fatty acids from the fat deposits, thus depriving the liver of its substrate for the production of ketones. ${ }^{11,12}$ This could occur when a new medication is prescribed to a child on the ketogenic diet or when drugs are given to this child for an acute illness.

The control of the insulin release by glucose is important for patients on the ketogenic diet. Both a high basal serum glucose level and an elevated peak level can increase the magnitude of subsequent insulin release for several hours. ${ }^{12}$ This is a possible explanation for the prolonged loss of ketosis seen when patients on the ketogenic diet ingest an excessive amount of carbohydrates. When this occurs, returning to ketosis may take 24 hours or more, since the insulin response to subsequent carbohydrate or amino acid exposure with the next meals may be greater. This "break" in ketosis often offsets seizure control. A short period (12 to 24 hours) of fasting may be necessary for a quick return to the ketotic state, but seizure control might not be regained. ${ }^{5}$ Clinicians should select, whenever it is possible, medications that will not affect the ketogenic diet. If a drug with a significant number of calories provided by carbohydrates must be selected, the diet should be adjusted accordingly. The data that has been published to help the clinician make these decisions are either out of date or compiled in the United States. ${ }^{9}$ Canadian clinicians now have a tool to help them select a formulation that is less likely to affect the diet. If this list fails to provide information needed by the clinician, the worst case scenario can be used to evaluate the caloric content of a drug. As it always overestimates the caloric content of a drug, we feel the table is safe to use.

\section{Drug-diet interactions.}

One must also be aware of unfavorable interactions between drugs and the diet. Initially, because of its broad spectrum of efficacy and also because it is a branched chain fatty acid, valproic acid was believed to be able to replace the diet with a lesser requirement of family training and teaching. ${ }^{6}$ This has not been the case. In fact, a number of significant interactions exist between valproic acid and the diet. One of its metabolites, 2propylpentanoyl-CoA-(valproyl-CoA) has been implicated in the inhibition of mitochondrial fatty acid oxidation. ${ }^{12}$ Valproic acid may also interfere with beta-oxidation of medium-chain fatty acids. ${ }^{13}$ This may be due to a direct action of 2-n-propyl-4pentenoic acid. ${ }^{13}$ Clinically, we have not found valproic acid to 
significantly interfere with ketogenesis in children on the diet but valproic acid may increase the risk of side effects in patients on the ketogenic diet. ${ }^{14}$ In younger children, we recommend the use of the capsule form of divalproex, available in the US under the brand name of Depakote, because it contains far less carbohydrates than the liquid formulation. ${ }^{15}$

Phenobarbital should be used with caution because serum concentration can increase as much as $100 \%$ over the baseline when the diet is started. ${ }^{16}$ Phenobarbital elimination is slowed down in acidotic state. Other anticonvulsant drugs that can directly affect ketosis include drugs that can reduce insulin release, such as phenytoin, ${ }^{17}$ and acetazolamide with high glucose concentrations. ${ }^{18}$ Other anticonvulsant drugs can increase insulin release like phenobarbital ${ }^{19}$ and acetazolamide with low glucose concentrations. ${ }^{18}$ Finally, one needs to be careful when using beta-blocking agents. Beta-blocking agents inhibit fatty acid and gluconeogenetic substrate release and reduce plasma glucagon levels. ${ }^{20}$ Patients on both beta-blocking agents and a diet low in carbohydrates and protein, or those undergoing fasting, are potentially more susceptible to hypoglycemia with decreased capability of ketogenesis. Betablocking agents may also decrease the symptoms of hypoglycemia. We therefore avoid such treatment when possible, especially during early stages of treatment.

In conclusion, we believe the information compiled in the tables represents a useful tool for the professionals involved in ketogenic diet programs. We also want to reinforce the need to be aware of the many possible interactions between drugs (anticonvulsants and others) and the mechanisms involved in the maintenance of ketosis.

\section{REFERENCES}

1. Freeman JM, Vining EP, Pillas DJ, et al. The efficacy of the ketogenic diet-1998: a prospective evaluation of intervention in 150 children. Pediatrics 1998;102:1358-1363.

2. Barboka CJ. Epilepsy in adults: results of treatment by ketogenic diet in one hundred cases. Arch Neurol Psychiatr 1930;23:904-914.

3. Guelpa G, Marie A. La lutte contre l'épilepsie par la désintoxication et par la rééducation alimentaire. Revue de Thérapie Medico-
Chirurgicale 1911;78:8-13.

4. Wilder RM. The effect of ketonemia on the course of epilepsy. Mayo Clin Bull 1921;2:(307);314.

5. Livingston SL. Comprehensive Management of Epilepsy in Infants, Childhood and Adolescence. Springfield, IL: Charles C Thomas. 1972:378-405.

6. Wheless JW. The ketogenic diet: fact or fiction. J Child Neurol 1995; 10:419-423.

7. Huttenlocher PR. Ketonemia and seizures: metabolic and anticonvulsant effects of two ketogenic diets in childhood epilepsy. Pediatr Res 1976;10(5):536-540.

8. Bough KJ, Matthews PJ, Eagles DA. A ketogenic diet has different effects upon seizures induced by maximal electroshock and by pentylenetetrazole infusion. Epilepsy Res 2000;38:105-114.

9. Thio LL, Wong M, Yamada KA. Ketone bodies do not directly alter excitatory or inhibitory hippocampal synaptic transmission. Neurology 2000;54:325-331.

10. Feldstein TJ. Carbohydrate and alcohol content of 200 oral liquid medications for use in patients receiving ketogenic diets. Pediatrics 1996;97:506-511.

11. Tallian KB, Nahata MC, Tsao CY. Role of the ketogenic diet with intractable seizures. Ann Pharmacother 1998;32:349-361.

12. McGarry JD. Glucose-fatty acid interactions in health and disease. Am J Clin Nutr 1998;67(Suppl):500S-504S.

13. Li J, Norwood DL, Mao LF, Schulz H. Mitochondrial metabolism of valproic acid. Biochemistry 1991;30:388-394.

14. Bjorge SM, Baillie TA. Inhibition of medium-chain fatty acid betaoxidation in vitro by valproic acid and its unsaturated metabolite, 2-n-propyl-4-pentenoic acid. Biochem Biophys Res Commun 1985; 132:245-252.

15. Ballaban-Gil K, Callahan C, O'Dell C, et al. Complications of the ketogenic diet. Epilepsia 1998;39:744-748.

16. Kinsman SL, Vining EPG, Quakey SA, Mellitis D, Freeman JM. Efficacy of the ketogenic diet for intractable seizure disorders: review of 58 cases. Epilepsia 1992;33:1132-1136.

17. Kizer JS, Vargas-Gordon M, Brendel K, Bressler R. The in vitro inhibition of insulin secretion by diphenylhydantoin. J Clin Invest 1970;49:1942-1948.

18. Boquist L, Backman AM, Stromberg C. Hyperglycemia produced in mice by administration of acetazolamide and diphenylhydantoin. Eur J Pharmacol 1980;64:325-332.

19. Venkatesan N, Davidson MB, Simsolo RB, Kern PA. Phenobarbital treatment enhances insulin-mediated glucose metabolism and improves lipid metabolism in the diabetic rat. Metabolism 1994;43:348-356.

20. Karam JH. Reversible insulin resistance in noninsulin-dependent diabetes mellitus. Horm Metab Res 1996;28:440-444. 
Table 1: Caloric content of drugs, listed by generic names

Drug, concentration and presentation

5-aminosalicylic acid, $500 \mathrm{mg} /$ Tablet

Acebutolol HCl, $100 \mathrm{mg} /$ Tablet

Acebutolol HCl, $200 \mathrm{mg} /$ Tablet

Acebutolol HCl, $400 \mathrm{mg} /$ Tablet

Acetaminophen, $16 \mathrm{mg} / \mathrm{ml} /$ Liquid

Acetaminophen, $16 \mathrm{mg} / \mathrm{ml} /$ Liquid

Acetaminophen, $16 \mathrm{mg} / \mathrm{ml} /$ Liquid

Acetaminophen, $160 \mathrm{mg} /$ Chewable tablet

Acetaminophen, $160 \mathrm{mg} /$ Chewable tablet

Acetaminophen, $32 \mathrm{mg} / \mathrm{ml} /$ Liquid

Acetaminophen, $32 \mathrm{mg} / \mathrm{ml} /$ Liquid

Acetaminophen, $32 \mathrm{mg} / \mathrm{ml} /$ Liquid

Acetaminophen, $32 \mathrm{mg} / \mathrm{ml} /$ Liquid

Acetaminophen, $32 \mathrm{mg} / \mathrm{ml} /$ Liquid

Acetaminophen, $325 \mathrm{mg} /$ Tablet

Acetaminophen, $325 \mathrm{mg} /$ Tablet

Acetaminophen, $500 \mathrm{mg} /$ Tablet

Acetaminophen, $500 \mathrm{mg} /$ Tablet

Acetaminophen, $80 \mathrm{mg} /$ Chewable tablet

Acetaminophen, $80 \mathrm{mg} /$ Chewable tablet

Acetaminophen, $80 \mathrm{mg} / \mathrm{ml} /$ Liquid

Acetaminophen, $80 \mathrm{mg} / \mathrm{ml} /$ Liquid

Acetaminophen, $80 \mathrm{mg} / \mathrm{ml} /$ Liquid

Acetaminophen, $80 \mathrm{mg} / \mathrm{ml} /$ Liquid

Acetaminophen, $80 \mathrm{mg} / \mathrm{ml} /$ Liquid

Acetaminophen-caffeine-codeine phosphate (C15), 300 mg-15 mg-15 mg / Tablet

Acetaminophen-caffeine-codeine phosphate (C30), 300 mg-15 mg-30 mg / Tablet

Acetaminophen-caffeine-codeine phosphate (C8), $300 \mathrm{mg}-15 \mathrm{mg}-8 \mathrm{mg} /$ Tablet

Acetazolamide, $250 \mathrm{mg} /$ Tablet

Acetazolamide, $250 \mathrm{mg} /$ Tablet

Acetazolamide, $250 \mathrm{mg} /$ Tablet

Acetylsalicylic acid, $325 \mathrm{mg} /$ Tablet

Acetylsalicylic acid, $650 \mathrm{mg} /$ Tablet

Acyclovir, $40 \mathrm{mg} / \mathrm{ml} /$ Liquid

Alfacalcidol, 0,2 mcg/ml / Liquid

Alfacalcidol, 0,25 mcg / Capsule

Alfacalcidol, $1 \mathrm{mcg} /$ Capsule

Allopurinol, $100 \mathrm{mg} /$ Tablet

Allopurinol, $200 \mathrm{mg} /$ Tablet

Allopurinol, $300 \mathrm{mg} /$ Tablet

Alprazolam, 0,25 mg / Tablet

Alprazolam, 0,50 mg / Tablet

Aluminium + magnesium hydroxyde, $45 \mathrm{mg}+40 \mathrm{mg} /$ Liquid

Aluminium + magnesium hydroxyde, / Liquid

Aluminium hydroxyde, $64 \mathrm{mg} / \mathrm{ml} /$ Liquid

Amantadin, $10 \mathrm{mg} / \mathrm{ml} /$ Liquid

Amiloride $\mathrm{HCl} / \mathrm{Hydrochlorothiazide,} \mathrm{5/50} \mathrm{mg} \mathrm{/} \mathrm{Tablet}$
Commercial name and company

\section{Caloric content* \\ $-\mathrm{CH}^{\S}$ (Kcal)}

Pentasa (Hoechst Marion Roussel)

Pentasa (Hoechst Marion Roussel)

Novo-Acebutol (Novopharm)

Novo-Acebutol (Novopharm)

Novo-Acebutol (Novopharm)

Tempra (Mead Johnson)

Atasol (Carter Horner)

Pms-acetaminophen (Pharmascience)

Tylenol (McNeil)

Tylenol sans sucrose (McNeil)

Tempra (Mead Johnson)

Tylenol elixir (McNeil)

Tylenol suspension liquid (grape) (McNeil)

Tylenol suspension liquid (bubblegum) (McNeil)

Pms-acetaminophen (Pharmascience)

Tylenol (McNeil)

Novo-Gesic (Novopharm)

Tylenol (McNeil)

Novo-Gesic (Novopharm)

Tylenol (McNeil)

Tylenol sans sucrose (McNeil)

Tempra (Mead Johnson)

Tylenol drops (McNeil)

Tylenol suspension liquid (McNeil)

Pms-acetaminophen (Pharmascience)

Atasol drops (Carter Horner)

Novo-Gesic C15 (Novopharm)

Novo-Gesic C30 (Novopharm)

Novo-Gesic C8 (Novopharm)

Apo-Acetazolamide (Apotex)

Diamox (Berlex)

Novo-Zolamide (Novopharm)

Novasen Novasen SP.C. (Novopharm)

Novasen Novasen SP.C. (Novopharm)

Zovirax (Glaxo Wellcome)

One-Alpha (Leo Pharma)

One-Alpha (Leo Pharma)

One-Alpha (Leo Pharma)

Novo-Purol (Novopharm)

Novo-Purol (Novopharm)

Novo-Purol (Novopharm)

Novo-Alprazol (Novopharm)

Novo-Alprazol (Novopharm)

Maalox (Novartis Pharma)

Almagel (Atlas laboratories)

Amphogel (Axcan Pharma)

Symmetrel (Du Pont Pharma)

Novamilor (Novopharm)

\begin{tabular}{ll}
0,00 & $\mathrm{Nd}$ \\
0,00 & $\mathrm{Nd}$ \\
0,360 & 0,375 \\
0,720 & 0,749 \\
0,025 & 0,066 \\
$\mathrm{Nd}$ & 1,78 \\
1,44 & 2,40 \\
1,7 & $\mathrm{Nd}$ \\
$\mathrm{Nd}$ & 2,5 \\
$\mathrm{Nd}$ & 2,0 \\
$\mathrm{Nd}$ & 1,78 \\
$\mathrm{Nd}$ & 1,76 \\
$\mathrm{Nd}$ & 2,22 \\
1,7 & 2,40 \\
$\mathrm{Nd}$ & $\mathrm{Nd}$ \\
0,039 & 0,2 \\
$\mathrm{Nd}$ & $\mathrm{Nd}$ \\
\hline 0,062 & 0,3 \\
\hline $\mathrm{Nd}$ & $\mathrm{Nd}$ \\
$\mathrm{Nd}$ & 1,2 \\
$\mathrm{Nd}$ & 1 \\
$\mathrm{Nd}$ & 1,40 \\
$\mathrm{Nd}$ & 0,98 \\
\hline 0,36 & 2,10 \\
\hline 1,79 & $\mathrm{Nd}$ \\
\hline & 2,40 \\
\hline
\end{tabular}

$0,148 \quad 0,188$

0,154

0,195

0,145

0,184

Nd $\quad 0,79$

$\mathrm{Nd} \quad 1,0$

$0,100 \quad 0,143$

$0,239 \quad \mathrm{Nd}$

$0,479 \quad \mathrm{Nd}$

$1,26 \quad 2,62$

$2,63 \quad \mathrm{Nd}$

$1,15 \quad \mathrm{Nd}$

$1,15 \quad \mathrm{Nd}$

$0,458 \quad 0,484$

$0,057 \quad 0,084$

$0,346 \quad 0,395$

$0,416 \quad 0,450$

$0,413 \quad 0,482$

$\mathrm{Nd} \quad 0,04$

$0,20 \quad \mathrm{Nd}$

$\mathrm{Nd} \quad 0,60$

$2,58 \quad \mathrm{Nd}$

0,401 
$\begin{array}{cc}\text { Caloric content* } & \text { Caloric content* } \\ - \text { CH§ (Kcal) } & - \text { Total (Kcal) }\end{array}$

- Total (Kcal)

Aminocaproic acid, $250 \mathrm{mg} / \mathrm{ml} /$ Liquid

Amicar(Wyeth-Ayerst)

Novo-Triptyn (Novopharm)

Amitriptylin, $10 \mathrm{mg} /$ Tablet

Amitriptylin, $25 \mathrm{mg} /$ Tablet

Amitriptylin, 2 mg/ml / Liquid

Amitriptylin, $50 \mathrm{mg} /$ Tablet

Amoxicillin + clavulanic acid, $50+12,5 \mathrm{mg} / \mathrm{ml} /$ Liquid

Novo-Triptyn (Novopharm)

Elavil (Merck Sharp \& Dohme)

Novo-Triptyn (Novopharm)

Clavulin (Smithkline Beecham Pharma)

Amoxicillin + potassium clavulanate, $25+6,25 \mathrm{mg} / \mathrm{ml} /$ Liquid $\quad$ Clavulin (SmithKline Beecham Pharma)

Amoxicillin + potassium clavulanate, $250 \mathrm{mg}+125 \mathrm{mg} /$ Tablet Clavulin (SmithKline Beecham Pharma)

Amoxicillin + clavulanate de potassium, $500 \mathrm{mg}+125 \mathrm{mg} / \mathrm{Tab}$

Amoxicillin, $125 \mathrm{mg} /$ Chewable tablet

Amoxicillin, $25 \mathrm{mg} / \mathrm{ml} /$ Liquid

Amoxicillin, $25 \mathrm{mg} / \mathrm{ml} /$ Liquid

Amoxicillin, $25 \mathrm{mg} / \mathrm{ml} /$ Liquid

Amoxicillin, $25 \mathrm{mg} / \mathrm{ml} /$ Liquid

Amoxicillin, $25 \mathrm{mg} / \mathrm{ml} /$ Liquid

Amoxicillin, 25 mg/ml / Liquid

Amoxicillin, 250 mg / Capsule

Amoxicillin, $250 \mathrm{mg} /$ Capsule

Amoxicillin, 250 mg / Capsule

Amoxicillin, $250 \mathrm{mg} /$ Capsule

Amoxicillin, $250 \mathrm{mg} /$ Chewable tablet

Amoxicillin, $50 \mathrm{mg} / \mathrm{ml} /$ Liquid

Amoxicillin, $50 \mathrm{mg} / \mathrm{ml} /$ Liquid

Amoxicillin, 50 mg/ml / Liquid

Amoxicillin, $50 \mathrm{mg} / \mathrm{ml} /$ Liquid

Amoxicillin, 50 mg/ml / Liquid

Amoxicillin, $50 \mathrm{mg} / \mathrm{ml} /$ Liquid

Amoxicillin, 500 mg / Capsule

Amoxicillin, 500 mg / Capsule

Amoxicillin, 500 mg / Capsule

Amoxicillin, 500 mg / Capsule

Ampicillin, 100 mg/ml / Liquid

Ampicillin, $25 \mathrm{mg} / \mathrm{ml} /$ Liquid

Ampicillin, $25 \mathrm{mg} / \mathrm{ml} /$ Liquid

Ampicillin, 25 mg/ml / Liquid

Ampicillin, $250 \mathrm{mg} /$ Capsule

Ampicillin, 250 mg / Capsule

Ampicillin, $250 \mathrm{mg} /$ Capsule

Ampicillin, $50 \mathrm{mg} / \mathrm{ml} /$ Liquid

Ampicillin, 50 mg/ml / Liquid

Ampicillin, $50 \mathrm{mg} / \mathrm{ml} /$ Liquid

Ampicillin, 500 mg / Capsule

Ampicillin, 500 mg / Capsule

Ampicillin, $500 \mathrm{mg} /$ Capsule

ASA-Caffeine-Codeine phosphate, 1 tablet / Tablet

Clavulin (SmithKline Beecham Pharma)

Amoxil(Wyeth-Ayerst)

Amoxil(Wyeth-Ayerst)

Apo-Amoxi hypoglucidic (Apotex)

Novamoxin hypoglucidic (Novopharm)

Nu-amoxi (Nu-Pharm)

Apo-amoxi (Apotex)

Novamoxin (Novopharm)

Amoxil(Wyeth-Ayerst)

Apo-Amoxi (Apotex)

Nu-Amoxi (Nu-Pharm)

Novamoxin (Novopharm)

Amoxil(Wyeth-Ayerst)

Amoxil(Wyeth-Ayerst)

Apo-Amoxi hypoglucidic (Apotex)

Novamoxin hypoglucidic (Novopharm)

Apo-amoxi (Apotex)

Nu-amoxi (Nu-Pharm)

Novamoxin (Novopharm)

Amoxil(Wyeth-Ayerst)

Apo-Amoxi (Apotex)

Nu-Amoxi (Nu-Pharm)

Novamoxin (Novopharm)

Novo-Ampicillin (Novopharm)

$\mathrm{Nd} \quad 2,40$

$0,039 \quad 0,065$

$0,046 \quad 0,063$

$2,63 \quad \mathrm{Nd}$

$0,082 \quad 0,138$

$0,18 \quad \mathrm{Nd}$

$0,18 \quad \mathrm{Nd}$

$1,20 \quad \mathrm{Nd}$

$1,80 \quad \mathrm{Nd}$

$\mathrm{Nd} \quad 0,50$

$\mathrm{Nd} \quad 0,40$

$\mathrm{Nd} \quad 0,026$

$0,48 \quad \mathrm{Nd}$

$1,78 \quad \mathrm{Nd}$

$1,79 \quad \mathrm{Nd}$

2,27 $\mathrm{Nd}$

$\mathrm{Nd} \quad 0,20$

$\mathrm{Nd} \quad 0,21$

$\mathrm{Nd} \quad 0,21$

$0,082 \quad 0,126$

$\mathrm{Nd} \quad 1,0$

$\mathrm{Nd} \quad 0,32$

$\mathrm{Nd} \quad 0,026$

$0,41 \quad \mathrm{Nd}$

$1,78 \quad \mathrm{Nd}$

$1,78 \quad \mathrm{Nd}$

$2,14 \quad \mathrm{Nd}$

$\mathrm{Nd} \quad 0,40$

$\mathrm{Nd} \quad 0,36$

$\mathrm{Nd} \quad 0,36$

$0,164 \quad 0,245$

Apo-ampi (Apotex)

Nu-ampi (Nu-Pharm)

1,87

$\mathrm{Nd}$

Novo-Ampicillin (Novopharm)

Apo-Ampi (Apotex)

Nu-Ampi (Nu-Pharm)

Novo-Ampicillin (Novopharm)

Apo-ampi (Apotex)

Nu-ampi (Nu-Pharm)

Novo-Ampicillin (Novopharm)

Apo-Ampi (Apotex)

Nu-Ampi (Nu-Pharm)

Novo-Ampicillin (Novopharm)

Novo A.C. \& C. (Novopharm)

Novo-Atenol (Novopharm)

Novo-Atenol (Novopharm)

Zithromax (Pfizer)

Zithromax (Pfizer)

Zithromax (Pfizer)

Zithromax (Pfizer)

Zithromax (Pfizer)

Penglobe (Astra Pharma)

Penglobe (Astra Pharma)

$\mathrm{Nd}$

2,27

$\mathrm{Nd}$

$\mathrm{Nd}$

0,104

$\mathrm{Nd}$

$\mathrm{Nd}$

2,16

$\mathrm{Nd}$

$\mathrm{Nd}$

0,078

0,067

0,188

0,094

38,828

3,15

0,928

3,16

0,348

$\mathrm{Nd}$

$\mathrm{Nd}$

1,87

1,82

1,82

2,27

0,22

0,22

0,148

1,70

1,70

2,16

0,39

0,39

0,155

0,067

0,222

0,111

$\mathrm{Nd}$

$\mathrm{Nd}$

$\mathrm{Nd}$

$\mathrm{Nd}$

Aacampicillin, chlorhydrate, $400 \mathrm{mg} /$ Tablet

Bacampicillin, chlorhydrate, $800 \mathrm{mg} /$ Tablet

.

$\mathrm{Nd}$
0,10
0,20 
LE JOURNAL CANADIEN DES SCIENCES NEUROLOGIQUES

\section{Caloric content* Caloric content* - CH§ (Kcal) - Total (Kcal)}

Baclofen, $20 \mathrm{mg} /$ Tablet

Benzydamine, 0,15\% / Liquid

Bisacodyl, $5 \mathrm{mg} /$ Dragee

Bisacodyl, $5 \mathrm{mg} /$ Tablet

Bisacodyl, $5 \mathrm{mg} /$ Tablet

Bismuth subsalicylate, $17,6 \mathrm{mg} / \mathrm{ml} /$ Liquid

Bromazepam, $3 \mathrm{mg} /$ Tablet

Bumetanide, $1 \mathrm{mg} /$ Tablet

Bumetanide, $2 \mathrm{mg} /$ Tablet

Bumetanide, $5 \mathrm{mg} /$ Tablet

Buspirone, $10 \mathrm{mg} /$ Tablet

Calcitriol, $1 \mathrm{mcg} / \mathrm{ml} /$ Liquid

Calcium gluconate + glucoheptonate, $20 \mathrm{mg} / \mathrm{ml} /$ Liquid

Calcium lactobionate, / Liquid

Calcium, 500 mg Ca elem. / Capsule

Captopril, $100 \mathrm{mg} /$ Tablet

Captopril, 12,5 mg / Tablet

Captopril, $25 \mathrm{mg} /$ Tablet

Captopril, $50 \mathrm{mg} /$ Tablet

Carbamazepine, $100 \mathrm{mg}$ / Chewable tablet

Carbamazepine, $20 \mathrm{mg} / \mathrm{ml} /$ Liquid

Carbamazepine, $200 \mathrm{mg} /$ Tablet

Carbamazepine, $200 \mathrm{mg} /$ Tablet

Carbamazepine, $200 \mathrm{mg} /$ Controled release tablet

Carbamazepine, $200 \mathrm{mg} /$ Tablet

Carbamazepine, $200 \mathrm{mg} /$ Tablet

Carbamazepine, $200 \mathrm{mg} /$ Controled release tablet

Carbamazepine, $400 \mathrm{mg}$ / Controled release tablet

Carnitine, $100 \mathrm{mg} / \mathrm{ml} /$ Liquid

Cefaclor, $25 \mathrm{mg} / \mathrm{ml} /$ Liquid

Cefaclor, $250 \mathrm{mg} /$ Capsule

Cefaclor, $250 \mathrm{mg} /$ Capsule

Cefaclor, $50 \mathrm{mg} / \mathrm{ml} /$ Liquid

Cefaclor, $500 \mathrm{mg} /$ Capsule

Cefaclor, $500 \mathrm{mg} /$ Capsule

Cefaclor, $75 \mathrm{mg} / \mathrm{ml} /$ Liquid

Cefixime, $20 \mathrm{mg} / \mathrm{ml} /$ Liquid

Cefixime, $400 \mathrm{mg} /$ Tablet

Cefprozil, $25 \mathrm{mg} / \mathrm{ml} /$ Liquid

Cefprozil, $250 \mathrm{mg} /$ Tablet

Cefprozil, $50 \mathrm{mg} / \mathrm{ml} /$ Liquid

Cefprozil, $500 \mathrm{mg} /$ Tablet

Cefuroxime axetil, $25 \mathrm{mg} / \mathrm{ml} /$ Liquid

Cefuroxime axetil, $250 \mathrm{mg} /$ Tablet

Cefuroxime axetil, $500 \mathrm{mg} /$ Tablet

Cephalexin, $25 \mathrm{mg} / \mathrm{ml} /$ Liquid

Cephalexin, $25 \mathrm{mg} / \mathrm{ml} /$ Liquid

Cephalexin, $25 \mathrm{mg} / \mathrm{ml} /$ Liquid

Cephalexin, $250 \mathrm{mg} /$ Capsule

Cephalexin, $250 \mathrm{mg} /$ Tablet

Cephalexin, $250 \mathrm{mg} /$ Tablet

Cephalexin, $250 \mathrm{mg} /$ Tablet

Cephalexin, $250 \mathrm{mg} /$ Tablet

Cephalexin, $50 \mathrm{mg} / \mathrm{ml} /$ Liquid
Novo-Baclofen (Novopharm)

Novo-Benzydamine (Novopharm)

Dulcolax (Boehringer Ingelheim)

Apo-Bisacodyl (Apotex)

Soflax EX (Pharmascience)

Pepto-bismol (Procter \& Gamble)

Novo-Bromazepam (Novopharm)

Burinex (Leo Pharma)

Burinex (Leo Pharma)

Burinex (Leo Pharma)

Novo-Buspirone (Novopharm)

Rocaltrol (Hoffman-La Roche)

Calcium Stanley (Stanley Pharmaceuticals)

Calcium-Sandoz (Sandoz)

Calsan (Novartis Sante Familiale)

Novo-Captopril (Novopharm)

Novo-Captopril (Novopharm)

Novo-Captopril (Novopharm)

Novo-Captopril (Novopharm)

Tegretol (Novartis Pharma)

Tegretol (Novartis Pharma)

Apo-Carbamazepine (Apotex)

Nu-Carbamazepine (Nu-Pharm)

Tegretol CR (Novartis Pharma)

Tegretol (Novartis Pharma)

Novo-Carbamaz (Novopharm)

Tegretol (Novartis Pharma)

Tegretol CR (Novartis Pharma)

Carnitor (Sigma-Tau Pharmaceuticals)

Ceclor (Eli Lilly)

Pms-Cefaclor (Pharmascience)

Apo-Cefaclor (Apotex)

Ceclor (Eli Lilly)

Pms-Cefaclor (Pharmascience)

Apo-Cefaclor (Apotex)

Ceclor (Eli Lilly)

Suprax (Rhône-Poulenc Rorer)

Suprax (Rhône-Poulenc Rorer)

Cefzil (Bristol-Myers Squibb)

Cefzil (Bristol-Myers Squibb)

Cefzil (Bristol-Myers Squibb)

Cefzil (Bristol-Myers Squibb)

Ceftin (Glaxo Wellcome)

Ceftin (Glaxo Wellcome)

Ceftin (Glaxo Wellcome)

Keflex (Eli Lilly)

Novo-Lexin (Novopharm)

Pms-cephalexin (Pharmascience)

Novo-Lexin (Novopharm)

Apo-Cephalex (Apotex)

$\mathrm{Nu}$-Cephalex (Nu-Pharm)

Novo-Lexin (Novopharm)

Pms-cephalexin (Pharmascience)

Keflex (Eli Lilly)
$0,132 \quad 0,156$

$0,808 \quad \mathrm{Nd}$

$\mathrm{Nd} \quad 0,26$

$\mathrm{Nd} \quad 0,48$

$0,12 \quad \mathrm{Nd}$

$1,00 \quad \mathrm{Nd}$

$0,400 \quad 0,417$

$0,61 \quad \mathrm{Nd}$

$0,74 \quad \mathrm{Nd}$

$1,17 \quad \mathrm{Nd}$

$0,487 \quad 0,499$

$8,60 \quad \mathrm{Nd}$

$\mathrm{Nd} \quad 0,04$

$\mathrm{Nd} \quad 1,35$

$\mathrm{Nd} \quad 5,85$

$0,311 \quad 0,329$

$0,039 \quad 0,041$

$0,078 \quad 0,082$

$0,156 \quad 0,165$

$\mathrm{Nd} \quad 1,08$

$\mathrm{Nd} \quad 1,79$

$\mathrm{Nd} \quad 0,35$

$\mathrm{Nd} \quad 0,35$

$\mathrm{Nd} \quad 0$

$0,00 \quad \mathrm{Nd}$

$0,031 \quad 0,048$

$\mathrm{Nd} \quad 2,12$

$\mathrm{Nd} \quad 0$

$0,20 \quad \mathrm{Nd}$

$\mathrm{Nd} \quad 2,52$

$0,14 \quad \mathrm{Nd}$

$0,28 \quad \mathrm{Nd}$

$\mathrm{Nd} \quad 2,42$

$0,28 \quad \mathrm{Nd}$

$0,317 \quad \mathrm{Nd}$

$\mathrm{Nd} \quad 2,24$

$2,22 \quad \mathrm{Nd}$

$0,396 \quad \mathrm{Nd}$

$\mathrm{Nd} \quad 1,50$

$0,064 \quad \mathrm{Nd}$

$\mathrm{Nd} \quad 1,30$

$0,128 \quad \mathrm{Nd}$

$2,45 \quad 2,59$

$0,00 \quad \mathrm{Nd}$

$0,00 \quad \mathrm{Nd}$

Nd 2,48

$2,32 \quad 2,32$

$2,48 \quad \mathrm{Nd}$

$0,044 \quad 0,109$

$\mathrm{Nd} \quad 0,51$

$\mathrm{Nd} \quad 0,51$

$0,075 \quad 0,104$

$0,51 \quad \mathrm{Nd}$

$\mathrm{Nd} \quad 2,42$

048

2

d

2

d

(

24

d

30

2,59

d

Nd

, 51

104


Caloric content* Caloric content* - CH§ (Kcal) - Total (Kcal)

Cephalexin, $50 \mathrm{mg} / \mathrm{ml} /$ Liquid

Cephalexin, $50 \mathrm{mg} / \mathrm{ml} /$ Liquid

Cephalexin, $500 \mathrm{mg} /$ Capsule

Cephalexin, $500 \mathrm{mg} /$ Tablet

Cephalexin, $500 \mathrm{mg} /$ Tablet

Cephalexin, $500 \mathrm{mg} /$ Tablet

Cephalexin, $500 \mathrm{mg} /$ Tablet

Chloral hydrate, $100 \mathrm{mg} / \mathrm{ml} /$ Liquid

Chloral hydrate, $500 \mathrm{mg} /$ Capsule

Chloramphenicol, $250 \mathrm{mg}$ / Capsule

Chlordiazepoxide, $10 \mathrm{mg} /$ Capsule

Chlordiazepoxide, $25 \mathrm{mg} /$ Capsule

Chlordiazepoxide, $5 \mathrm{mg} /$ Capsule

Chloroquine, $250 \mathrm{mg} /$ Tablet

Chlorpheniramine, $4 \mathrm{mg} /$ Tablet

Chlorpromazine, $20 \mathrm{mg} / \mathrm{ml}$ / Liquid

Chlorpromazine, $5 \mathrm{mg} / \mathrm{ml} /$ Liquid

Chlorpromazine, $10 \mathrm{mg} /$ Tablet

Chlorpromazine, $100 \mathrm{mg} /$ Tablet

Chlorpromazine, $20 \mathrm{mg} / \mathrm{ml} /$ Liquid

Chlorpromazine, $200 \mathrm{mg} /$ Tablet

Chlorpromazine, $25 \mathrm{mg} /$ Tablet

Chlorpromazine, $40 \mathrm{mg} / \mathrm{ml}$ / Liquid

Chlorpromazine, $40 \mathrm{mg} / \mathrm{ml} /$ Liquid

Chlorpromazine, $50 \mathrm{mg} /$ Tablet

Chlorpropamide, $250 \mathrm{mg} /$ Tablet

Chlorthalidone, $100 \mathrm{mg} /$ Tablet

Chlorthalidone, $50 \mathrm{mg} /$ Tablet

Cholestyramine, 4 g/dose / Powder

Cholestyramine, 4 g/dose / Powder

Cimetidine, $200 \mathrm{mg} /$ Tablet

Cimetidine, $300 \mathrm{mg} /$ Tablet

Cimetidine, $400 \mathrm{mg} /$ Tablet

Cimetidine, $600 \mathrm{mg} /$ Tablet

Cimetidine, $800 \mathrm{mg} /$ Tablet

Ciprofloxacin, $250 \mathrm{mg} /$ Tablet

Ciprofloxacin, $500 \mathrm{mg} /$ Tablet

Ciprofloxacin, chlorhydrate, $100 \mathrm{mg} / \mathrm{ml} /$ Liquid

Cisapride, $1 \mathrm{mg} / \mathrm{ml} /$ Liquid

Clarithromycin, $250 \mathrm{mg} /$ Tablet

Clarithromycin, $25 \mathrm{mg} / \mathrm{ml} /$ Liquid

Clarithromycin, $500 \mathrm{mg} /$ Tablet

Clindamycin, palmitate, $15 \mathrm{mg} / \mathrm{ml} /$ Liquid

Clobazam, $10 \mathrm{mg} /$ Tablet

Clomipramine $\mathrm{HCl}, 10 \mathrm{mg} /$ Tablet

Clomipramine $\mathrm{HCl}, 25 \mathrm{mg} /$ Tablet

Clomipramine $\mathrm{HCl}, 50 \mathrm{mg} /$ Tablet

Clonazepam, 0,25 mg / Tablet

Clonazepam, 0,5 mg / Tablet

Clonazepam, 0,5 mg / Tablet

Clonazepam, 0,5 mg / Tablet

Clonazepam, 0,5 mg / Tablet

Clonazepam, 0,5 mg / Tablet

Clonazepam, $1 \mathrm{mg} /$ Tablet

\begin{tabular}{|c|c|c|}
\hline Novo-Lexin (Novopharm) & 2,23 & 2,23 \\
\hline Pms-cephalexin (Pharmascience) & 2,42 & $\mathrm{Nd}$ \\
\hline Novo-Lexin (Novopharm) & 0,088 & 0,211 \\
\hline Apo-Cephalex (Apotex) & $\mathrm{Nd}$ & 1,02 \\
\hline Nu-Cephalex (Nu-Pharm) & $\mathrm{Nd}$ & 1,02 \\
\hline Novo-Lexin (Novopharm) & 0,150 & 0,213 \\
\hline Pms-cephalexin (Pharmascience) & 1,02 & $\mathrm{Nd}$ \\
\hline Pms-chloralhydrate (Pharmascience) & 2,0 & $\mathrm{Nd}$ \\
\hline Novo-Chlorhydrate (Novopharm) & 0,00 & 0,060 \\
\hline Novo-Chlorocap (Novopharm) & 0,863 & 0,932 \\
\hline Novo-Poxide SP.C. (Novopharm) & 0,465 & 0,476 \\
\hline Novo-Poxide (Novopharm) & 0,416 & 0,426 \\
\hline Novo-Poxide (Novopharm) & 0,482 & 0,492 \\
\hline Novo-Chloroquine (Novopharm) & 0,824 & 0,988 \\
\hline Novo-pheniram (Novopharm) & 0,032 & 0,051 \\
\hline Largactil (Rhône-Poulenc Rorer) & 2,86 & $\mathrm{Nd}$ \\
\hline Largactil (Rhône-Poulenc Rorer) & 3,04 & $\mathrm{Nd}$ \\
\hline Novo-Chlorpromazine (Novopharm) & 0,064 & 0,094 \\
\hline Novo-Chlorpromazine (Novopharm) & 0,216 & 0,243 \\
\hline Chlorpromanyl (Technilab) & $\mathrm{Nd}$ & 3,40 \\
\hline Novo-Chlorpromazine (Novopharm) & 0,479 & 0,568 \\
\hline Novo-Chlorpromazine (Novopharm) & 0,124 & 0,139 \\
\hline Chlorpromanyl (Technilab) & $\mathrm{Nd}$ & 3,60 \\
\hline Largactil (Rhône-Poulenc Rorer) & 0,78 & $\mathrm{Nd}$ \\
\hline Novo-Chlorpromazine (Novopharm) & 0,114 & 0,132 \\
\hline Novo-Propamide (Novopharm) & 0,933 & 0,978 \\
\hline Novo-Thalidone (Novopharm) & 0,037 & 0,049 \\
\hline Novo-Thalidone (Novopharm) & 0,037 & 0,049 \\
\hline Novo-Cholamine Light (Novopharm) & 0,00 & 0,00 \\
\hline Novo-Cholamine (Novopharm) & 16,09 & 16,09 \\
\hline Novo-Cimetidine (Novopharm) & 0,053 & 0,075 \\
\hline Novo-Cimetidine (Novopharm) & 0,059 & 0,093 \\
\hline Novo-Cimetidine (Novopharm) & 0,106 & 0,150 \\
\hline Novo-Cimetidine (Novopharm) & 0,159 & 0,226 \\
\hline Novo-Cimetidine (Novopharm) & 0,211 & 0,301 \\
\hline Cipro (Bayer) & 0,171 & $\mathrm{Nd}$ \\
\hline Cipro (Bayer) & 0,333 & $\mathrm{Nd}$ \\
\hline Cipro (Bayer) & 6,40 & $\mathrm{Nd}$ \\
\hline Prepulsid (Jansen-Ortho) & 0,80 & $\mathrm{Nd}$ \\
\hline Biaxin (Abbott Laboratories) & 0,21 & $\mathrm{Nd}$ \\
\hline Biaxin pediatric (Abbott Laboratories) & 2,35 & $\mathrm{Nd}$ \\
\hline Biaxin (Abbott Laboratories) & 0,00 & $\mathrm{Nd}$ \\
\hline Dalacin C (Pharmacia \& Upjohn) & $\mathrm{Nd}$ & 1,20 \\
\hline Frisium (Hoechst Marion Roussel) & 0,398 & $\mathrm{Nd}$ \\
\hline Novo-Clopamine (Novopharm) & 0,209 & 0,212 \\
\hline Novo-Clopamine (Novopharm) & 0,00 & 0,003 \\
\hline Novo-Clopamine (Novopharm) & 0,00 & 0,009 \\
\hline Pms-Clonazepam (Pharmascience) & 0,26 & $\mathrm{Nd}$ \\
\hline Rivotril (Hoffman-La Roche) & $\mathrm{Nd}$ & 0,6 \\
\hline Pms-Clonazepam (Pharmascience) & 0,13 & $\mathrm{Nd}$ \\
\hline Apo-Clonazepam (Apotex) & 0,48 & $\mathrm{Nd}$ \\
\hline Clonapam (ICN) & 0,72 & $\mathrm{Nd}$ \\
\hline Rho-Clonazepam (Rhodiapharm) & 0,72 & $\mathrm{Nd}$ \\
\hline Pms-Clonazepam (Pharmascience) & 0,26 & $\mathrm{Nd}$ \\
\hline
\end{tabular}


LE JOURNAL CANADIEN DES SCIENCES NEUROLOGIQUES

Drug, concentration and presentation

Commercial name and company

Caloric content* Caloric content*

- CH§ (Kcal)

- Total (Kcal)

Clonazepam, 1,0 mg / Tablet

Clonazepam, 1,0 mg / Tablet

Clonapam (ICN)

$0,725 \quad \mathrm{Nd}$

Clonazepam, $2 \mathrm{mg} /$ Tablet

Clonazepam, $2 \mathrm{mg} /$ Tablet

Clonazepam, $2 \mathrm{mg} /$ Tablet

Clonazepam, 2,0 mg / Tablet

Clonazepam, 2,0 mg / Tablet

Clonidin, 0,1 mg / Tablet

Clonidin, 0,2 mg / Tablet

Clorazepate, $15 \mathrm{mg} /$ Capsule

Clorazepate, 3,75 mg / Capsule

Clorazepate, 7,5 mg / Capsule

Cloxacillin sodium, $250 \mathrm{mg} /$ Capsule

Rho-Clonazepam (Rhodiapharm)

Rivotril (Hoffman-La Roche)

Pms-Clonazepam (Pharmascience)

$0,725 \quad \mathrm{Nd}$

Rho-Clonazepam (Rhodiapharm)

Apo-Clonazepam (Apotex)

$\mathrm{Nd} \quad 0,6$

Cloxacillin sodium, $250 \mathrm{mg} /$ Capsule

Cloxacillin sodium, $500 \mathrm{mg} /$ Capsule

Cloxacillin sodium, $500 \mathrm{mg} /$ Capsule

Cloxacillin, $25 \mathrm{mg} / \mathrm{ml} /$ Liquid

Cloxacillin, $25 \mathrm{mg} / \mathrm{ml} /$ Liquid

Cloxacillin, $25 \mathrm{mg} / \mathrm{ml} /$ Liquid

Cloxacillin, $25 \mathrm{mg} / \mathrm{ml} /$ Liquid

Cloxacillin, $250 \mathrm{mg} /$ Capsule

Cloxacillin, $250 \mathrm{mg} /$ Capsule

Cloxacillin, $500 \mathrm{mg} /$ Capsule

Cloxacillin, $500 \mathrm{mg} /$ Capsule

Clonapam (ICN)

$0,54 \quad \mathrm{Nd}$

0.722

Novo-Clonidine (Novopharm)

$0,4 \quad \mathrm{Nd}$

Novo-Clonidine (Novopharm)

0,722

Novo-Clopate (Novopharm)

Novo-Clopate (Novopharm)

Novo-Clopate (Novopharm)

Apo-Cloxi (Apotex)

$\mathrm{Nu}-\mathrm{Cloxi}$ (Nu-Pharm)

Apo-Cloxi (Apotex)

Nu-Cloxi (Nu-Pharm)

Apo-Cloxi (Apotex)

Nu-Cloxi (Nu-Pharm)

0,418

0,796

0,601

0,475

0,463

$\mathrm{Nd}$

Orbenine (Wyeth-Ayerst)

Novo-Cloxin (Novopharm)

Orbenine (Wyeth-Ayerst)

Novo-Cloxin (Novopharm)

Orbenine (Wyeth-Ayerst)

Novo-Cloxin (Novopharm)

Codeine phosphate (Rougier)

Codeine, $5 \mathrm{mg} / \mathrm{ml} /$ Liquid

Cyclobenzaprine $\mathrm{HCl}, 10 \mathrm{mg} /$ Tablet

Novo-Cycloprine (Novopharm)

Neoral (Sandoz)

Cyclosporine, $100 \mathrm{mg} / \mathrm{ml} /$ Liquid

Cyproheptadine, 0,4 mg/ml / Liquid

Dapsone, $100 \mathrm{mg} /$ Tablet

Periactin (Johnson \& Johnson Merck)

Avlosulfon(Wyeth-Ayerst)

Demeclocycline, chlorhydrate, $150 \mathrm{mg}$ / Tablet

Demeclocycline, chlorhydrate, $300 \mathrm{mg} /$ Tablet

Declomycin(Wyeth-Ayerst)

Declomycin(Wyeth-Ayerst)

Novo-Desipramine (Novopharm)

$\mathrm{Nd}$

$\mathrm{Nd}$

$\mathrm{Nd} \quad 0,33$

$\mathrm{Nd} \quad 1,79$

$\mathrm{Nd} \quad 1,79$

Desipramine $\mathrm{HCl}, 10 \mathrm{mg} /$ Tablet

Desipramine HCl, $25 \mathrm{mg}$ / Tablet

Desipramine $\mathrm{HCl}, 50 \mathrm{mg} /$ Tablet

Desipramine HCl, 75 mg / Tablet

Desogestrel-ethinyl estradiol, 0,5 mg / Tablet

Desogestrel-ethinyl estradiol, $1 \mathrm{mg} /$ Capsule

Desogestrel-ethinyl estradiol, $2 \mathrm{mg} /$ Capsule

Dexamethasone, $1 \mathrm{mg} / \mathrm{ml} /$ Liquid

Dexamethasone, $4 \mathrm{mg} /$ Tablet

Dexamethasone, $500 \mathrm{mg} /$ Tablet

Dexamethasone, $750 \mathrm{mg} /$ Tablet

Novo-Desipramine (Novopharm)

Novo-Desipramine (Novopharm)

Novo-Desipramine (Novopharm)

Marvelon (Organon.)

Marvelon (Organon.)

$\mathrm{Nd} \quad 2,00$

1,80

2,00

$\mathrm{Nd}$

0,139

1,80

Marvelon (Organon.)

$\mathrm{Nd}$

0,30

0,123

$\mathrm{Nd} \quad 3,33$

$0,533 \quad 0,542$

$7,00 \quad \mathrm{Nd}$

$1,80 \quad \mathrm{Nd}$

$\mathrm{Nd} \quad 0,12$

$\mathrm{Nd} \quad 1,0$

Pms-dexamethasone (Pharmascience)

Dexasone (ICN)

Dexasone (ICN)

Dexasone (ICN)

$\mathrm{Nd} \quad 1,0$

$0,003 \quad 0,005$

0,007

0,010

0,010

0,281

0,281

0,281

0,64

0,591

0,591

0,589

0,012

Dextrometorphan, bromhydrate

Sugar free, $3 \mathrm{mg} / \mathrm{ml}$ / Liquid

Balminil DM (Rougier)

1,40

0,019

0,018

$\mathrm{Nd}$

$\mathrm{Nd}$

$\mathrm{Nd}$

$\mathrm{Nd}$

$\mathrm{Nd}$

$\mathrm{Nd}$

$\mathrm{Nd}$

Dextrometorphan, bromhydrate - pseudoephedrine - guaifenesin,

$3 \mathrm{mg}-6 \mathrm{mg}-20 \mathrm{mg} / \mathrm{ml} /$ Liquid

Novahistex DM Expt Dcgt (Hoechst Marion Roussel) 2,60

$\mathrm{Nd}$

Dextrometorphan, bromhydrate - pseudoephedrine, $1,5 \mathrm{mg}-3 \mathrm{mg} / \mathrm{ml} /$ Liquid

Novahistine DM Dcgt (Hoechst Marion Roussel) 3,00

$\mathrm{Nd}$

Dextrometorphan, bromhydrate - pseudoephedrine, $3 \mathrm{mg}-6 \mathrm{mg} / \mathrm{ml} /$ Liquid

Dextrometorphan, bromhydrate, $3 \mathrm{mg} / \mathrm{ml}$ / Liquid

Dextrometorphan, bromhydrate, $3 \mathrm{mg} / \mathrm{ml}$ / Liquid

Dextrometorphan, bromhydrate de - pseudoephedrine -

guaifenesin, $1,5 \mathrm{mg}-3 \mathrm{mg}-10 \mathrm{mg} / \mathrm{ml} /$ Liquid

Novahistex DM Dcgt (Hoechst Marion Roussel)

Balminil DM (Rougier)

2,40

2,40

Novahistex DM (Hoechst Marion Roussel)

2,40

$\mathrm{Nd}$

Novahistine DM Expt Dcgt (Hoechst Marion Roussel)2,44

$\mathrm{Nd}$

$\mathrm{Nd}$

$\mathrm{Nd}$

Nd




\section{Caloric content* Caloric content*} - CH§ (Kcal) - Total (Kcal)

Dextrometorphan, bromhydrate, $1,5 \mathrm{mg} / \mathrm{ml} /$ Liquid

Diazepam, $1 \mathrm{mg} / \mathrm{ml} /$ Liquid

Diazepam, $10 \mathrm{mg} /$ Tablet

Diazepam, $10 \mathrm{mg} /$ Tablet

Diazepam, $2 \mathrm{mg} /$ Tablet

Diazepam, $2 \mathrm{mg} /$ Tablet

Diazepam, $2 \mathrm{mg} /$ Tablet

Diazepam, $5 \mathrm{mg} /$ Tablet

Diazepam, 5 mg / Tablet

Diazepam, $5 \mathrm{mg} /$ Tablet

Diclofenac, $100 \mathrm{mg} /$ Tablet

Diclofenac, 25 mg / Tablet

Diclofenac, $50 \mathrm{mg} /$ Tablet

Diclofenac, 75 mg / Tablet

Dicyclomine, $10 \mathrm{mg} /$ Tablet

Dicyclomine, $2 \mathrm{mg} / \mathrm{ml}$ / Liquid

Dicyclomine, $20 \mathrm{mg} /$ Tablet

Diflunisal, 250 mg / Tablet

Diflunisal, $500 \mathrm{mg} /$ Tablet

Digoxin, 0,05 mg/ml / Liquid

Diltiazem, $120 \mathrm{mg} /$ Tablet

Diltiazem, $30 \mathrm{mg} /$ Tablet

Diltiazem, $60 \mathrm{mg} /$ Tablet

Diltiazem, $60 \mathrm{mg} /$ Tablet

Diltiazem, $90 \mathrm{mg} /$ Tablet

Dimenhydrinate, $15 \mathrm{mg} /$ Tablet

Dimenhydrinate, $15 \mathrm{mg} /$ Chewable tablet

Dimenhydrinate, $25 \mathrm{mg} /$ Tablet

Dimenhydrinate, $25 \mathrm{mg}$ immediate + $50 \mathrm{mg}$ slow release/Capsule Gravol (Carter Horner)

Dimenhydrinate, $3 \mathrm{mg} / \mathrm{ml} /$ Liquid

Dimenhydrinate, $3 \mathrm{mg} / \mathrm{ml} /$ Liquid

Dimenhydrinate, $50 \mathrm{mg} /$ Tablet

Dimenhydrinate, $50 \mathrm{mg} /$ Chewable tablet

Diphenhydramine, $2,5 \mathrm{mg} / \mathrm{ml} /$ Liquid

Diphenhydramine, $2,5 \mathrm{mg} / \mathrm{ml} /$ Liquid

Diphenhydramine, $6,25 \mathrm{mg} / \mathrm{ml} /$ Liquid

Dipyridamole, $100 \mathrm{mg} /$ Tablet

Dipyridamole, 25 mg / Tablet

Dipyridamole, $50 \mathrm{mg} /$ Tablet

Dipyridamole, $75 \mathrm{mg} /$ Tablet

Divalproex sodium, $125 \mathrm{mg} /$ Tablet

Divalproex sodium, $250 \mathrm{mg} /$ Tablet

Divalproex sodium, $500 \mathrm{mg} /$ Tablet

Docusate calcium, $240 \mathrm{mg} /$ Capsule

Docusate, calcium, $240 \mathrm{mg} /$ Capsule

Docusate, calcium, $240 \mathrm{mg} /$ Capsule

Docusa, sodium + casanthranol, $100 \mathrm{mg}+30 \mathrm{mg} /$ Capsule

Docusate, sodium + sennosides, $50 \mathrm{mg}+8,6 \mathrm{mg} /$ Tablet

Docusate, sodium, $10 \mathrm{mg} / \mathrm{ml} /$ Liquid

Docusate, sodium, $10 \mathrm{mg} / \mathrm{ml} /$ Liquid

Docusate, sodium, $100 \mathrm{mg} /$ Capsule

Docusate, sodium, $100 \mathrm{mg} /$ Capsule

Docusate, sodium, $100 \mathrm{mg} /$ Capsule

Docusate, sodium, $200 \mathrm{mg} /$ Capsule

\begin{tabular}{|c|c|c|}
\hline Novahistine DM (Hoechst Marion Roussel) & 3,00 & $\mathrm{Nd}$ \\
\hline Pms-diazepam (Pharmascience) & 1,38 & $\mathrm{Nd}$ \\
\hline Apo-Diazepam (Apotex) & $\mathrm{Nd}$ & 0,59 \\
\hline Valium Roche Oral (Hoffman-La Roche) & $\mathrm{Nd}$ & 0,7 \\
\hline Apo-Diazepam (Apotex) & $\mathrm{Nd}$ & 0,62 \\
\hline Vivol (Carter Horner) & $\mathrm{Nd}$ & 0,4 \\
\hline Novo-Dipam (Novopharm) & 0,710 & 0,744 \\
\hline Apo-Diazepam (Apotex) & $\mathrm{Nd}$ & 0,61 \\
\hline Valium Roche Oral (Hoffman-La Roche) & $\mathrm{Nd}$ & 0,7 \\
\hline Novo-Dipam (Novopharm) & 0,695 & 0,712 \\
\hline Novo-Difenac SR (Novopharm) & 0,878 & 0,900 \\
\hline Novo-Difenac (Novopharm) & 0,129 & 0,135 \\
\hline Novo-Difenac (Novopharm) & 0,109 & 0,116 \\
\hline Novo-Difenac SR (Novopharm) & 0,658 & 0,675 \\
\hline Bentylol (Hoechst Marion Roussel) & 0,369 & $\mathrm{Nd}$ \\
\hline Bentylol (Hoechst Marion Roussel) & 4,00 & $\mathrm{Nd}$ \\
\hline Bentylol (Hoechst Marion Roussel) & 0,369 & $\mathrm{Nd}$ \\
\hline Novo-Diflunisal (Novopharm) & 0,125 & 0,155 \\
\hline Novo-Diflunisal (Novopharm) & 0,250 & 0,311 \\
\hline Lanoxin (Glaxo Wellcome) & 1,93 & 2,30 \\
\hline Novo-Diltazem SR (Novopharm) & 0,00 & 0,00 \\
\hline Novo-Diltazem (Novopharm) & 0,623 & 0,641 \\
\hline Novo-Diltazem SR (Novopharm) & 0,00 & 0,00 \\
\hline Novo-Diltazem (Novopharm) & 1,246 & 1,282 \\
\hline Novo-Diltazem SR (Novopharm) & 0,00 & 0,00 \\
\hline Gravol (Carter Horner) & $\mathrm{Nd}$ & 0,20 \\
\hline Gravol (Carter Horner) & $\mathrm{Nd}$ & 1,40 \\
\hline Gravol (Carter Horner) & $\mathrm{Nd}$ & 0,30 \\
\hline Gravol (Carter Horner) & & 1,20 \\
\hline Gravol (Carter Horner) & $\mathrm{Nd}$ & 2,94 \\
\hline Pms-dimenhydrinate (Pharmascience) & 1,5 & $\mathrm{Nd}$ \\
\hline Gravol (Carter Horner) & $\mathrm{Nd}$ & 1,40 \\
\hline Gravol (Carter Horner) & $\mathrm{Nd}$ & 2,60 \\
\hline Benadryl elixir (Warner-Lambert) & $\mathrm{Nd}$ & 2,04 \\
\hline Pms-diphenydramine (Pharmascience) & 1,7 & $\mathrm{Nd}$ \\
\hline Benadryl (Warner-Lambert) & $\mathrm{Nd}$ & 1,57 \\
\hline Novo-Dipiradol (Novopharm) & 0,088 & 0,479 \\
\hline Novo-Dipiradol (Novopharm) & 0,022 & 0,153 \\
\hline Novo-Dipiradol (Novopharm) & 0,044 & 0,293 \\
\hline Novo-Dipiradol (Novopharm) & 0,066 & 0,379 \\
\hline Epival (Abbot) & 0,08 & $\mathrm{Nd}$ \\
\hline Epival (Abbot) & 0,17 & $\mathrm{Nd}$ \\
\hline Epival (Abbot) & 0,33 & $\mathrm{Nd}$ \\
\hline Surfak (Hoechst Marion Roussel) & 0,555 & $\mathrm{Nd}$ \\
\hline Pms-Docusate Calcium (Pharmascience) & 1,00 & $\mathrm{Nd}$ \\
\hline Soflax C (Pharmascience) & 1,00 & $\mathrm{Nd}$ \\
\hline Peri-Colace (Roberts Pharmaceutical) & 1,00 & $\mathrm{Nd}$ \\
\hline Senokot-S (Purdue Frederick) & $\mathrm{Nd}$ & 0,11 \\
\hline Colace (Roberts Pharmaceutical) & $\mathrm{Nd}$ & 2,40 \\
\hline Pms-docusate sodium (Pharmascience) & 1,7 & $\mathrm{Nd}$ \\
\hline Pms-docusate sodium (Pharmascience) & 0,51 & $\mathrm{Nd}$ \\
\hline Soflax (Pharmascience) & 0,51 & $\mathrm{Nd}$ \\
\hline Colace (Roberts Pharmaceutical) & 1,00 & $\mathrm{Nd}$ \\
\hline Soflax (Pharmascience) & 0,51 & $\mathrm{Nd}$ \\
\hline
\end{tabular}


LE JOURNAL CANADIEN DES SCIENCES NEUROLOGIQUES

Docusate, sodium, $4 \mathrm{mg} / \mathrm{ml}$ / Liquid

Docusate, sodium, $4 \mathrm{mg} / \mathrm{ml}$ / Liquid

Docusate, sodium, $4 \mathrm{mg} / \mathrm{ml}$ / Liquid

Docusate, sodium, $4 \mathrm{mg} / \mathrm{ml} /$ Liquid

Docusate, sodium, $4 \mathrm{mg} / \mathrm{ml} /$ Liquid

Docusate, sodium, $50 \mathrm{mg} / \mathrm{ml} /$ Liquid

Doxepin $\mathrm{HCl}, 100 \mathrm{mg} /$ Capsule

Doxepin $\mathrm{HCl}, 150 \mathrm{mg} /$ Capsule

Doxepin HCl, 25 mg / Capsule

Doxepin $\mathrm{HCl}, 50 \mathrm{mg} /$ Capsule

Doxepin $\mathrm{HCl}, 75 \mathrm{mg} /$ Capsule

Doxycycline, hyclate, $100 \mathrm{mg} /$ Capsule

Doxycycline, hyclate, $100 \mathrm{mg} /$ Capsule

Doxycycline, hyclate, $100 \mathrm{mg}$ / Capsule

Doxycycline, hyclate, $100 \mathrm{mg} /$ Capsule

Doxycycline, hyclate, $100 \mathrm{mg} /$ Tablet

Doxycycline, hyclate, $100 \mathrm{mg} /$ Tablet

Doxycycline, hyclate, $100 \mathrm{mg} /$ Tablet

Erythromycin base, $25 \mathrm{mg} / \mathrm{ml}$ / Liquid

Erythromycin base, $250 \mathrm{mg} /$ Capsule

Erythromycin base, $250 \mathrm{mg}$ / Capsule

Erythromycin base, $250 \mathrm{mg} /$ Tablet

Erythromycin base, $250 \mathrm{mg} /$ Tablet

Erythromycin base, $333 \mathrm{mg} /$ Capsule

Erythromycin base, $333 \mathrm{mg} /$ Capsule

Erythromycin base, $333 \mathrm{mg} /$ Tablet

Erythromycin base, $50 \mathrm{mg} / \mathrm{ml} /$ Liquid

Erythromycin base, $500 \mathrm{mg} /$ Tablet

Erythromycin, estolate, $25 \mathrm{mg} / \mathrm{ml} /$ Liquid

Erythromycin, estolate, $50 \mathrm{mg} / \mathrm{ml} /$ Liquid

Erythromycin, estolate, $25 \mathrm{mg} / \mathrm{ml} /$ Liquid

Erythromycin, estolate, $250 \mathrm{mg} /$ Capsule

Erythromycin, estolate, $50 \mathrm{mg} / \mathrm{ml} /$ Liquid

Erythromycin, stearate, $25 \mathrm{mg} / \mathrm{ml} /$ Liquid

Erythromycin, stearate, $250 \mathrm{mg} /$ Tablet

Erythromycin, stearate, $250 \mathrm{mg} /$ Tablet

Erythromycin, stearate, $250 \mathrm{mg} /$ Tablet

Erythromycin, stearate, $50 \mathrm{mg} / \mathrm{ml} /$ Liquid

Erythromycin, stearate, $500 \mathrm{mg} /$ Tablet

Erythromycin, succinate, $40 \mathrm{mg} / \mathrm{ml} /$ Liquid

Erythromycin, succinate, $600 \mathrm{mg} /$ Tablet

Erythromycin, succinate, $600 \mathrm{mg} /$ Tablet

Erythromycin, succinate, $80 \mathrm{mg} / \mathrm{ml} /$ Liquid

Erythromycin, succinate, $40 \mathrm{mg} / \mathrm{ml} /$ Liquid

Erythromycin, succinate, $80 \mathrm{mg} / \mathrm{ml} /$ Liquid

Erythromycine-sulfisoxazole, $40-120 \mathrm{mg} / \mathrm{ml}$ / Liquid

Ethosuximide, $50 \mathrm{mg} / \mathrm{ml} /$ Liquid

Famotidine, $10 \mathrm{mg} /$ Tablet

Famotidine, $20 \mathrm{mg} /$ Tablet

Famotidine, $40 \mathrm{mg} /$ Tablet

Fenofibrate, $100 \mathrm{mg} /$ Capsule

Ferrous sulfate, $15 \mathrm{mg}$ (element. iron)/ml / Liquid

Ferrous sulfate, $15 \mathrm{mg}$ (element. iron)/ml / Liquid

Ferrous sulfate, $300 \mathrm{mg} /$ Tablet

\begin{tabular}{|c|c|c|}
\hline Docusate de sodium (Technilab) & $\mathrm{Nd}$ & 2,78 \\
\hline Docusate Sodic (Taro Pharmaceuticals) & $\mathrm{Nd}$ & 2,40 \\
\hline Pms-docusate sodium (Pharmascience) & 1,7 & $\mathrm{Nd}$ \\
\hline Colace (Roberts Pharmaceutical) & 2,40 & $\mathrm{Nd}$ \\
\hline Docusate sodium syrup (Altas Laboratories) & 2,40 & $\mathrm{Nd}$ \\
\hline Pms-docusate sodium (Pharmascience) & 1,05 & $\mathrm{Nd}$ \\
\hline Novo-Doxepine (Novopharm) & 0,772 & 0,819 \\
\hline Novo-Doxepine (Novopharm) & 0,923 & 0,990 \\
\hline Novo-Doxepine (Novopharm) & 0,428 & 0,452 \\
\hline Novo-Doxepine (Novopharm) & 0,694 & 0,735 \\
\hline Novo-Doxepine (Novopharm) & 0,568 & 0,608 \\
\hline Apo-Doxy (Apotex) & $\mathrm{Nd}$ & 0,74 \\
\hline Nu-Doxycycline (Nu-Pharm) & $\mathrm{Nd}$ & 0,74 \\
\hline Novo-Doxylin (Novopharm) & 0,022 & 0,052 \\
\hline Vibramycin (Pfizer) & 0,956 & $\mathrm{Nd}$ \\
\hline Apo-Doxy (Apotex) & $\mathrm{Nd}$ & 0,44 \\
\hline Nu-Doxycycline (Nu-Pharm) & $\mathrm{Nd}$ & 0,44 \\
\hline Vibra-Tabs (Pfizer) & 0,516 & $\mathrm{Nd}$ \\
\hline Erythrocin (Abbot) & $\mathrm{Nd}$ & 1,96 \\
\hline Apo-Erythro E.C. (Apotex) & $\mathrm{Nd}$ & 0,20 \\
\hline Novo-Rythro Encap (Novopharm) & 0,198 & $\mathrm{Nd}$ \\
\hline Apo-Erythro Base (Apotex) & $\mathrm{Nd}$ & 1,34 \\
\hline Erythromid (Abbott Laboratories) & 0,00 & $\mathrm{Nd}$ \\
\hline Eryc (Parke-Davis) & $\mathrm{Nd}$ & 0,13 \\
\hline Apo-Erythro E.C. (Apotex) & 0,473 & $\mathrm{Nd}$ \\
\hline PCE (Laboratoires Abbott Laboratories) & 0,64 & $\mathrm{Nd}$ \\
\hline Erythrocin (Abbot) & $\mathrm{Nd}$ & 1,84 \\
\hline Erybid (Abbott Laboratories) & 0,00 & $\mathrm{Nd}$ \\
\hline Ilosone (Eli Lilly) & $\mathrm{Nd}$ & 1,8 \\
\hline Ilosone (Eli Lilly) & $\mathrm{Nd}$ & 1,42 \\
\hline Novo-rythro estolate (Novopharm) & 1,37 & 1,37 \\
\hline Novo-Rythro Estolate (Novopharm) & 0,266 & 0,330 \\
\hline Novo-rythro estolate (Novopharm) & 1,37 & 1,37 \\
\hline Erythrocin (Abbott Laboratories) & $\mathrm{Nd}$ & 1,96 \\
\hline Apo-Erythro S (Apotex) & $\mathrm{Nd}$ & 0,87 \\
\hline Nu-Erythromycin-S (Nu-Pharm) & $\mathrm{Nd}$ & 0,87 \\
\hline Novo-Rythro Stearate (Novopharm) & 0,185 & 1,846 \\
\hline Erythrocin (Abbott Laboratories) & $\mathrm{Nd}$ & 1,84 \\
\hline Apo-Erythro S (Apotex) & $\mathrm{Nd}$ & 1,73 \\
\hline EES 200 (Abbott Laboratories) & 2,63 & $\mathrm{Nd}$ \\
\hline Apo-Erythro-ES (Apotex) & $\mathrm{Nd}$ & 1,39 \\
\hline EES 600 (Abbott Laboratories) & 0,17 & $\mathrm{Nd}$ \\
\hline EES 400 (Abbott Laboratories) & 2,43 & $\mathrm{Nd}$ \\
\hline Novo-rythro ethyl succinate (Novopharm) & 1,96 & 1,96 \\
\hline Novo-rythro ethyl succinate (Novopharm) & 1,76 & 1,76 \\
\hline Pediazole (Abbot) & $\mathrm{Nd}$ & 1,50 \\
\hline Zarontin (Parke-Davis) & $\mathrm{Nd}$ & 3,00 \\
\hline Novo-Famotidine (Novopharm) & 0,066 & 0,075 \\
\hline Novo-Famotidine (Novopharm) & 0,132 & 0,149 \\
\hline Novo-Famotidine (Novopharm) & 0,132 & 0,149 \\
\hline Novo-Fenofibrate (Novopharm) & 0,554 & 0,566 \\
\hline Pms-ferrous sulfate (Pharmascience) & 2,71 & $\mathrm{Nd}$ \\
\hline Fer-In-Sol (Mead Johnson) & 2,76 & $\mathrm{Nd}$ \\
\hline Apo-sulfate ferreux (Apotex) & $\mathrm{Nd}$ & 0,30 \\
\hline
\end{tabular}



$\begin{array}{cc}\text { Caloric content* } & \text { Caloric content* } \\ - \text { CH§ (Kcal) } & - \text { Total (Kcal) }\end{array}$

Ferrous sulfate, $300 \mathrm{mg}$ / Tablet

Ferrous sulfate, $6 \mathrm{mg}($ element. iron)/ml / Liquid

Ferrous sulfate, $6 \mathrm{mg}($ element. iron) $/ \mathrm{ml} /$ Liquid

Fluconazole, $10 \mathrm{mg} / \mathrm{ml} /$ Liquid

Flunarizine $\mathrm{HCl}, 5 \mathrm{mg} /$ Capsule

Fluorure sodium, $5,56 \mathrm{mg} / \mathrm{ml} /$ Liquid

Fluoxetine, $4 \mathrm{mg} / \mathrm{ml}$ / Liquid

Fluoxetine, $10 \mathrm{mg} /$ Capsule

Fluoxetine, $20 \mathrm{mg} /$ Capsule

Fluoxetine, $4 \mathrm{mg} / \mathrm{ml} /$ Liquid

Fluphenazine, $0,5 \mathrm{mg} / \mathrm{ml} /$ Liquid

Flurazepam, $15 \mathrm{mg} /$ Capsule

Flurazepam, $30 \mathrm{mg}$ / Capsule

Flurbiprofen, $100 \mathrm{mg} /$ Tablet

Flurbiprofen, $50 \mathrm{mg}$ / Tablet

Flutamide, $250 \mathrm{mg} /$ Tablet

Furosemide, $10 \mathrm{mg} / \mathrm{ml}$ / Liquid

Furosemide, $20 \mathrm{mg} /$ Tablet

Furosemide, $20 \mathrm{mg}$ / Tablet

Furosemide, $40 \mathrm{mg} /$ Tablet

Furosemide, $40 \mathrm{mg} /$ Tablet

Furosemide, $500 \mathrm{mg} /$ Tablet

Furosemide, $80 \mathrm{mg} /$ Tablet

Furosemide, $80 \mathrm{mg} /$ Tablet

Fusidic acid, $250 \mathrm{mg} /$ Tablet

Fusidic acid, 49,2 mg/ ml / Liquid

Fusidic acid, $50 \mathrm{mg} / \mathrm{ml} /$ Liquid

Gemfibrozil, 300 mg / Capsule

Gemfibrozil, $600 \mathrm{mg} /$ Capsule

Glyburide, 2,5 mg / Tablet

Glyburide, $5 \mathrm{mg} /$ Tablet

Grepafloxacin, chlorhydrate, $200 \mathrm{mg} /$ Tablet

Guaifenesine, $20 \mathrm{mg} / \mathrm{ml}$ / Liquid

Guaifenesin, $20 \mathrm{mg} / \mathrm{ml} /$ Liquid

Haloperidol, 0,5 mg / Tablet

Haloperidol, $1 \mathrm{mg} /$ Tablet

Haloperidol, 10 mg / Tablet

Haloperidol, 2 mg / Tablet

Haloperidol, $2 \mathrm{mg} / \mathrm{ml} /$ Liquid

Haloperidol, $2 \mathrm{mg} / \mathrm{ml} /$ Liquid

Haloperidol, $2 \mathrm{mg} / \mathrm{ml} /$ Liquid

Haloperidol, $20 \mathrm{mg} /$ Tablet

Haloperidol, $5 \mathrm{mg} /$ Tablet

Hydralazine $\mathrm{HCl}, 10 \mathrm{mg} /$ Tablet

Hydralazine, $25 \mathrm{mg} /$ Tablet

Hydralazine, $50 \mathrm{mg} /$ Tablet

Hydrochlorothiazide, $25 \mathrm{mg} /$ Tablet

Hydrochlorothiazide, $50 \mathrm{mg} /$ Tablet

Hydrochlorothiazide/methyldopa, 250/15 mg / Tablet

Hydrochlorothiazide/methyldopa, 250/25 mg / Tablet

Hydromorphone $\mathrm{HCl}, 1 \mathrm{mg} / \mathrm{ml} /$ Liquid

Hydromorphone $\mathrm{HCl}, 1 \mathrm{mg} / \mathrm{ml} /$ Liquid

Hydroxyzine, $2 \mathrm{mg} / \mathrm{ml}$ / Liquid

Hydroxyzin, $10 \mathrm{mg} /$ Capsule
Pms-sulfate ferreux (Pharmascience)

Pms-ferrous sulfate (Pharmascience)

Fer-In-Sol (Mead Johnson)

Diflucan (Pfizer)

Novo-Flunarizine (Novopharm)

Fluor-A-Day (Pharmascience)

Prozac (Eli Lilly)

Novo-Fluoxetine (Novopharm)

Novo-Fluoxetine (Novopharm)

Apo-fluoxetine (Apotex)

Pms-fluphenazine (Pharmascience)

Novo-Flupam / Novo-Flupam SP.C. (Novopharm) 1,193

Novo-Flupam / Novo-Flupam SP.C. (Novopharm)

Novo-Flurprofen (Novopharm)

Novo-Flurprofen (Novopharm)

Novo-Flutamide (Novopharm)

Lasix (Hoechst Marion Roussel)

Novo-Semide (Novopharm)

Lasix (Hoechst Marion Roussel)

Novo-Semide (Novopharm)

Lasix (Hoechst Marion Roussel)

Lasix special (Hoechst Marion Roussel)

Novo-Semide (Novopharm)

Lasix (Hoechst Marion Roussel)

Fucidin (Leo Pharma)

Fucidin (Leo Pharma)

Fucidin (Leo Pharma)

Novo-Gemfibrozil (Novopharm)

Novo-Gemfibrozil (Novopharm)

Novo-Glyburide (Novopharm)

Novo-Glyburide (Novopharm)

Raxar (Glaxo Wellcome)

Balminil E (Rougier)

Balminil E sugar free (Rougier)

Novo-Peridol (Novopharm)

Novo-Peridol (Novopharm)

Novo-Peridol (Novopharm)

Novo-Peridol (Novopharm)

Apo-haloperidol (Apotex)

Novo-peridol (Novopharm)

Pms-haloperidol (Pharmascience)

Novo-Peridol (Novopharm)

Novo-Peridol (Novopharm)

Novo-Hylazin (Novopharm)

Novo-Hylazin (Novopharm)

Novo-Hylazin (Novopharm)

Novo-Hydrazide (Novopharm)

Novo-Hydrazide (Novopharm)

Novo-Doparil-15 (Novopharm)

Novo-Doparil-25 (Novopharm)

Dilaudid (Knoll)

Pms-hydromorphone (Pharmascience)

Atarax (Pfizer)

Apo-Hydroxyzine (Apotex)
$0,57 \quad \mathrm{Nd}$

$1,22 \quad \mathrm{Nd}$

$5,00 \quad \mathrm{Nd}$

$2,72 \quad \mathrm{Nd}$

$0,576 \quad 0,583$

$0 \quad \mathrm{Nd}$

$\mathrm{Nd} \quad 2,40$

$0,730 \quad 0,747$

$0,694 \quad 0,710$

$3,64 \quad \mathrm{Nd}$

2,39 $\mathrm{Nd}$

1,236

1,103

0,401

0,322

1,311

$\mathrm{Nd}$

0,187

$\mathrm{Nd}$

0,377

$\mathrm{Nd}$

$\mathrm{Nd}$

0,751

$\mathrm{Nd}$

0,29

0,37

0,78

0,568

0,259

0,055

0,110

$\mathrm{Nd}$

1,60

$\mathrm{Nd} \quad 1,62$

$0,025 \quad 0,041$

$0,025 \quad 0,041$

$0,050 \quad 0,082$

0,025

$\mathrm{Nd}$

0,00

0,00

0,050

0,025

0,292

0,182

0,100

0,238

0,477

0,168

0,135

$\mathrm{Nd}$

1,09

$\mathrm{Nd}$

$\mathrm{Nd}$

3,20
0,47

0,041

0,01

0,00

0,00

0,082

0,041

0,301

0,189

0,106

0,247

0,495

0,211

0,178

2,00

$\mathrm{Nd}$

0,47

$\mathrm{Nd} \quad 1,60$


LE JOURNAL CANADIEN DES SCIENCES NEUROLOGIQUES

Hydroxyzine, $10 \mathrm{mg}$ / Capsule

Hydroxyzine, $2 \mathrm{mg} / \mathrm{ml} /$ Liquid

Hydroxyzine, $25 \mathrm{mg} /$ Capsule

Hydroxyzine, $25 \mathrm{mg} /$ Capsule

Hydroxyzine, $50 \mathrm{mg} /$ Capsule

Hydroxyzine, $50 \mathrm{mg} /$ Capsule

Ibuprofen, $20 \mathrm{mg} / \mathrm{ml} /$ Liquid

Ibuprofen, $200 \mathrm{mg}$ / Tablet

Ibuprofen, $200 \mathrm{mg} /$ Tablet (sugar coating)

Ibuprofen, $300 \mathrm{mg}$ / Tablet

Ibuprofen, $300 \mathrm{mg} /$ Tablet (sugar coating)

Ibuprofen, $400 \mathrm{mg}$ / Tablet

Ibuprofen, $400 \mathrm{mg} /$ Tablet (suger coating)

Ibuprofen, $600 \mathrm{mg} /$ Tablet

Imipramine, $10 \mathrm{mg} /$ Tablet

Imipramine, $25 \mathrm{mg} /$ Tablet

Imipramine, $50 \mathrm{mg} /$ Tablet

Indapamide, 2,5 mg / Tablet

Indomethacin, $25 \mathrm{mg} /$ Capsule

Indomethacin, $50 \mathrm{mg} /$ Capsule

Ioniazid, $10 \mathrm{mg} / \mathrm{ml} /$ Liquid

Isosorbide dinitrate, $10 \mathrm{mg} /$ Tablet

Isosorbide dinitrate, $30 \mathrm{mg} /$ Tablet

Itraconazole, $10 \mathrm{mg} / \mathrm{ml} /$ Liquid

Ketoconazole, $20 \mathrm{mg} / \mathrm{ml} /$ Liquid

Ketotifen, fumarate, $0,2 \mathrm{mg} / \mathrm{ml} /$ Liquid

Ketotifen, fumarate, $0,2 \mathrm{mg} / \mathrm{ml} /$ Liquid

Ketotifen, fumarate, $1 \mathrm{mg} /$ Tablet

Ketotifen, fumarate, $0,2 \mathrm{mg} / \mathrm{ml} /$ Liquid

Lactose (placebo) / Capsule

Lactulose, 666,7 mg/ml / Liquid

Lactulose, 666,7 mg/ml / Liquid

Lamivudine, $10 \mathrm{mg} / \mathrm{ml} /$ Liquid

Lamotrigine, $100 \mathrm{mg} /$ Tablet

Lamotrigine, $150 \mathrm{mg} /$ Tablet

Lamotrigine, $25 \mathrm{mg} /$ Tablet

Lidocaine, $2 \%$ / Liquid

Lithium, carbonate, $60 \mathrm{mg} / \mathrm{ml} /$ Liquid

Loperamide, 0,2 $\mathrm{mg} / \mathrm{ml} /$ Liquid

Loperamide, $2 \mathrm{mg} /$ Tablet

Loratadine, $1 \mathrm{mg} / \mathrm{ml} /$ Liquid

Lorazepam, 0,5 mg / Tablet

Lorazepam, 0,5 mg / Tablet (Sublingual)

Lorazepam, 0,5 mg / Tablet

Lorazepam, 0,5 mg / Tablet

Lorazepam, $1 \mathrm{mg} /$ Tablet

Lorazepam, 1,0 mg / Tablet

Lorazepam, 1,0 mg / Tablet (Sublingual)

Lorazepam, 1,0 mg / Tablet

Lorazepam, $2 \mathrm{mg} /$ Tablet

Lorazepam, 2,0 mg / Tablet

Lorazepam, 2,0 mg / Tablet (Sublingual)

Lorazepam, 2,0 mg / Tablet

Magaldrate, $96 \mathrm{mg} / \mathrm{ml} /$ Liquid
Novo-Hydroxyzin (Novopharm)

Pms-hydroxyzine (Pharmascience)

Apo-Hydroxyzine (Apotex)

Novo-Hydroxyzin (Novopharm)

Apo-Hydroxyzine (Apotex)

Novo-Hydroxyzin (Novopharm)

Advil (Whitehall-Robins)

Novo-Profen (Novopharm)

Novo-Profen (Novopharm)

Novo-Profen (Novopharm)

Novo-Profen (Novopharm)

Novo-Profen (Novopharm)

Novo-Profen (Novopharm)

Novo-Profen (Novopharm)

Novo-Pramine (Novopharm)

Novo-Pramine (Novopharm)

Novo-Pramine (Novopharm)

Novo-Indapamide (Novopharm)

Novo-Methacin / Novo-Methacin SP.

Novo-Methacin / Novo-Methacin SP.C.

Pms-isoniazid (Pharmascience) $\quad 0,89$

Novo-Sorbide (Novopharm) $\quad 0,160$

Novo-Sorbide (Novopharm) $\quad 0,420$

Sporanox (Jansen-Ortho) $\quad 0,50$

Nizoral (Jansen-Ortho)

0,25

Apo-Ketotifen (Apotex)

Novo-Ketotifen (Novopharm)

Novo-Ketotifen (Novopharm)

Zaditen (Novartis Pharma)

Novo-Plus (Novopharm)

Gen-lac (Genpharm)

Pms-lactulose (Pharmascience)

3-TC (Glaxo Wellcome)

Lamictal (Glaxo Wellcome)

Lamictal (Glaxo Wellcome)

Lamictal (Glaxo Wellcome)

Pms-lidocaine viscous (Pharmascience)

Pms-lithium citrate (Pharmascience)

Pms-loperamide (Pharmascience)

Novo-Loperamide (Novopharm)

Claritin (Schering)

Apo-Lorazepam (Apotex)

Ativan(Wyeth-Ayerst)

$\mathrm{Nu}$-Loraz (Nu-Pharm)

Novo-Lorazem (Novopharm)

Novo-Lorazem (Novopharm)

Apo-Lorazepam (Apotex)

Ativan (Wyeth-Ayerst)

Nu-Loraz (Nu-Pharm)

Novo-Lorazem (Novopharm)

Apo-Lorazepam (Apotex)

Ativan (Wyeth-Ayerst)

Nu-Loraz (Nu-Pharm)

Riopan (Whitehall-Robins)
1,40

$\mathrm{Nd}$

0,44

1,30

0,44

1,25

$\mathrm{Nd}$

0,215

0,291

0,322

0,425

0,429

0,532

0,593

0,088

0,088

0,209

0,260

0,910

1,495

$\mathrm{Nd}$

0,174

0,442

$\mathrm{Nd}$

$\mathrm{Nd}$

$\mathrm{Nd}$

3,31

3,31

0,493

2,41

0,720

$\mathrm{Nd}$

3,5

1,13

0,428

0,644

0,107

0,2

1,77

2,19

1,353

3,20

$\mathrm{Nd}$

$\mathrm{Nd}$

$\mathrm{Nd}$

0,049

0,098

$\mathrm{Nd}$

$\mathrm{Nd}$

Nd

0,110

Nd

$\mathrm{Nd}$

$\mathrm{Nd}$

0
0,499

$\mathrm{Nd}$

0,732

3,64

Nd

1,27

$\mathrm{Nd}$

$\mathrm{Nd}$

$\mathrm{Nd}$

$\mathrm{Nd}$

$\mathrm{Nd}$

$\mathrm{Nd}$

1,383

Nd

0,19

0,14

0,19

0,049

0,099

0,38

0,59

0,38

0,112

0,47

0,19

0,47

$\mathrm{Nd}$ 

$\begin{array}{cc}\text { Caloric content* } & \text { Caloric content* } \\ - \text { CH§ (Kcal) } & - \text { Total (Kcal) }\end{array}$

Magnesium, citrate, $15 \mathrm{~g} /$ flacon / Liquid

Magnesium, hydroxyde, $80 \mathrm{mg} / \mathrm{ml} /$ Liquid

Maprotiline $\mathrm{HCl}, 10 \mathrm{mg} /$ Tablet

Maprotiline $\mathrm{HCl}, 50 \mathrm{mg} /$ Tablet

Maprotiline $\mathrm{HCl}, 75 \mathrm{mg} /$ Tablet

Medroxyprogesterone acetate, $10 \mathrm{mg} /$ Tablet

Medroxyprogesterone acetate, 2,5 mg / Tablet

Medroxyprogesterone acetate, $5 \mathrm{mg} /$ Tablet

Meprobamate, $200 \mathrm{mg} /$ Tablet

Meprobamate, $400 \mathrm{mg} /$ Tablet

Methotrimeprazine maleate, $25 \mathrm{mg}$ / Tablet

Methotrimeprazine maleate, $50 \mathrm{mg} /$ Tablet

Methotrimeprazine, $40 \mathrm{mg} / \mathrm{ml} /$ Liquid

Methotrimeprazine, $5 \mathrm{mg} / \mathrm{ml}$ / Liquid

Methyldopa, $125 \mathrm{mg} /$ Tablet

Methyldopa, $250 \mathrm{mg} /$ Tablet

Methyldopa, $500 \mathrm{mg} /$ Tablet

Metoclopramide, $1 \mathrm{mg} / \mathrm{ml} /$ Liquid

Metoclopramide, chlorhydrate, $1 \mathrm{mg} / \mathrm{ml} /$ Liquid

Metoclopramide, chlorhydrate, $10 \mathrm{mg} /$ Tablet

Metoclopramide, chlorhydrate, $5 \mathrm{mg} /$ Tablet

Metoprolol, $100 \mathrm{mg} /$ Tablet coated

Metoprolol, $100 \mathrm{mg} /$ Tablet uncoated

Metoprolol, $50 \mathrm{mg} /$ Tablet coated

Metoprolol, $50 \mathrm{mg} /$ Tablet uncoated

Metronidazole, $250 \mathrm{mg} /$ Tablet

Metronidazole, $250 \mathrm{mg}$ / Tablet

Metronidazole, $500 \mathrm{mg} /$ Capsule

Mexiletine, chlorhydrate, $100 \mathrm{mg} /$ Granules

Mexiletine, chlorhydrate, $200 \mathrm{mg} /$ Granules

Mineral oil $78 \%$ sugar free jelly, / Jelly

Mineral oil $78 \%$ jelly, / Jelly

Mineral oil + glycerine, / Liquid

Minocycline, chlorhydrate, $100 \mathrm{mg} /$ Capsule

Minocycline, chlorhydrate, $100 \mathrm{mg}$ / Capsule

Minocycline, chlorhydrate, $50 \mathrm{mg} /$ Capsule

Minocycline, chlorhydrate, $50 \mathrm{mg} /$ Capsule

Minocycline, chlorhydrate, $100 \mathrm{mg} /$ Capsule

Minocycline, chlorhydrate, $50 \mathrm{mg} /$ Capsule

Morphine, $1 \mathrm{mg} / \mathrm{ml} /$ Liquid

Morphine, $1 \mathrm{mg} / \mathrm{ml}$ / Liquid

Morphine, $10 \mathrm{mg} / \mathrm{ml} /$ Liquid

Morphine, $10 \mathrm{mg} / \mathrm{ml} /$ Liquid

Morphine, $20 \mathrm{mg} / \mathrm{ml} /$ Liquid

Morphine, $5 \mathrm{mg} / \mathrm{ml} /$ Liquid

Morphine, $5 \mathrm{mg} / \mathrm{ml} /$ Liquid

Nadolol, $160 \mathrm{mg} /$ Tablet

Nadolol, $40 \mathrm{mg} /$ Tablet

Nadolol, $80 \mathrm{mg} /$ Tablet

Naproxen, 125 mg / Tablet

Naproxen, $25 \mathrm{mg} / \mathrm{ml} /$ Liquid

Naproxen, $250 \mathrm{mg} /$ Tablet

Naproxen, $375 \mathrm{mg} /$ Tablet

Naproxen, $500 \mathrm{mg} /$ Tablet

\begin{tabular}{|c|c|c|}
\hline Citro-Mag (Rougier) & $\mathrm{Nd}$ & 1,05 \\
\hline Lait de magnesie (Atlas laboratories) & 0 & $\mathrm{Nd}$ \\
\hline Novo-Maprotiline (Novopharm) & 0,290 & 0,299 \\
\hline Novo-Maprotiline (Novopharm) & 0,266 & 0,279 \\
\hline Novo-Maprotiline (Novopharm) & 0,366 & 0,384 \\
\hline Novo-Medrone (Novopharm) & 0,428 & 0,433 \\
\hline Novo-Medrone (Novopharm) & 0,380 & 0,384 \\
\hline Novo-Medrone (Novopharm) & 0,370 & 0,374 \\
\hline Novo-Mepro (Novopharm) & 0,084 & 0,117 \\
\hline Novo-Mepro (Novopharm) & 0,168 & 0,235 \\
\hline Novo-Meprazine (Novopharm) & 0,023 & 0,035 \\
\hline Novo-Meprazine (Novopharm) & 0,104 & 0,130 \\
\hline Nozinan (Rhône-Poulenc Rorer) & $\mathrm{Nd}$ & 1,80 \\
\hline Nozinan (Rhône-Poulenc Rorer) & $\mathrm{Nd}$ & 2,98 \\
\hline Novo-Medopa (Novopharm) & 0,060 & 0,074 \\
\hline Novo-Medopa (Novopharm) & 0,119 & 0,148 \\
\hline Novo-Medopa (Novopharm) & 0,238 & 0,297 \\
\hline Pms-metoclopramide (Pharmascience) & 1,3 & $\mathrm{Nd}$ \\
\hline Maxeran (Hoechst Marion Roussel) & 1,960 & $\mathrm{Nd}$ \\
\hline Maxeran (Hoechst Marion Roussel) & 0,361 & $\mathrm{Nd}$ \\
\hline Maxeran (Hoechst Marion Roussel) & 0,371 & $\mathrm{Nd}$ \\
\hline Novo-Metoprol (Novopharm) & 0,601 & 0,635 \\
\hline Novo-Metoprol Uncoated (Novopharm) & 0,601 & 0,631 \\
\hline Novo-Metoprol (Novopharm) & 0,509 & 0,531 \\
\hline Novo-Metoprol Uncoated (Novopharm) & 0,301 & 0,340 \\
\hline Apo-Metronidazole (Apotex) & $\mathrm{Nd}$ & 0,95 \\
\hline Novo-Nidazole (Novopharm) & 0,585 & 0,620 \\
\hline Flagyl (Rhône-Poulenc Rorer) & 0,00 & $\mathrm{Nd}$ \\
\hline Novo-Mexiletine (Novopharm) & 0,192 & 0,200 \\
\hline Novo-Mexiletine (Novopharm) & 0,383 & 0,401 \\
\hline Lansoyl (Jouveinal) & $\mathrm{Nd}$ & 0,27 \\
\hline Lansoyl (Jouveinal) & $\mathrm{Nd}$ & 0,60 \\
\hline Agarol (Warner-Lambert) & $\mathrm{Nd}$ & 0,65 \\
\hline Apo-Minocycline (Apotex) & $\mathrm{Nd}$ & 0,56 \\
\hline Minocin (Wyeth-Ayerst) & $\mathrm{Nd}$ & 1,0 \\
\hline Apo-Minocycline (Apotex) & $\mathrm{Nd}$ & 0,28 \\
\hline Minocin (Wyeth-Ayerst) & $\mathrm{Nd}$ & 1,0 \\
\hline Novo-Minocyline (Novopharm) & 0,608 & 0,625 \\
\hline Novo-Minocyline (Novopharm) & 0,524 & 0,536 \\
\hline Morphitec-1 (Technilab) & $\mathrm{Nd}$ & 2,23 \\
\hline M.O.S. (ICN) & 0,21 & $\mathrm{Nd}$ \\
\hline Morphitec-10 (Technilab) & $\mathrm{Nd}$ & 2,23 \\
\hline M.O.S. (ICN) & 1,00 & $\mathrm{Nd}$ \\
\hline Morphitec-20 (Technilab) & $\mathrm{Nd}$ & 2,23 \\
\hline Morphitec-5 (Technilab) & $\mathrm{Nd}$ & 2,23 \\
\hline M.O.S. (ICN) & 1,17 & $\mathrm{Nd}$ \\
\hline Novo-Nadolol (Novopharm) & 1,228 & 1,276 \\
\hline Novo-Nadolol (Novopharm) & 0,307 & 0,320 \\
\hline Novo-Nadolol (Novopharm) & 0,614 & 0,639 \\
\hline Novo-Naprox (Novopharm) & 0,037 & 0,061 \\
\hline Naprosyn (Hoffman-La Roche) & 1,16 & $\mathrm{Nd}$ \\
\hline Novo-Naprox (Novopharm) & 0,075 & 0,122 \\
\hline Novo-Naprox (Novopharm) & 0,112 & 0,183 \\
\hline Novo-Naprox (Novopharm) & 0,149 & 0,245 \\
\hline
\end{tabular}


LE JOURNAL CANADIEN DES SCIENCES NEUROLOGIQUES

Drug, concentration and presentation

Commercial name and company

Caloric content* Caloric content*

- CH§ (Kcal)

- Total (Kcal)

Naproxen, sodium, $275 \mathrm{mg}$ / Tablet

Novo-Naprox Sodium (Novopharm)

$0,077 \quad 0,135$

Naproxen, sodium, $550 \mathrm{mg} /$ Tablet

Novo-Naprox Sodium D.S. (Novopharm)

0,154

Nitrazepam, 10 mg / Table

Nitrazepam, 10 mg / Tablet

Nitrazepam, 10 mg / Tablet

Nitrazadon (ICN)

0,47

Rho-Nitrazepam (Rhodiapharm)

0,47

Mogadon (Hoffman-La Roche)

0,70

Nitrazepam, $5 \mathrm{mg} /$ Tablet

Nitrazadon (ICN)

0,233

Rho-Nitrazepam (Rhodiapharm)

0,233

Nitrazepam, $5 \mathrm{mg} /$ Tablet

Mogadon (Hoffman-La Roche)

0,35

Nitrofurantoin, $100 \mathrm{mg} /$ Tablet

Nitrofurantoin, $5 \mathrm{mg} / \mathrm{ml} /$ Liquid

Nitrofurantoin, $50 \mathrm{mg} /$ Capsule

Novo-Furan (Novopharm)

Novo-Furan (Novopharm)

0,146

Novo-Furantoin Capsules (Novopharm)

2,25

Nitrofurantoin, $50 \mathrm{mg} /$ Tablet

Norfloxacin, / Tablet

Novo-Furan (Novopharm)

0,037

Apo-Norflox (Apotex)

0,073

Norfloxacin, $400 \mathrm{mg} /$ Tablet

Normethadone + ephedrine, $10 \mathrm{mg} / \mathrm{ml} /$ Liquid

Noroxin (Merck Sharp \& Dohme)

0,044

Cophylac (Hoechst Marion Roussel)

0,00

Nystatin, 100000 u/ml / Liquid

Nystatin, $100000 \mathrm{u} / \mathrm{ml} /$ Liquid

Nilstat (Technilab)

Nystatin, $100000 \mathrm{u} / \mathrm{ml} /$ Liquid

Mycostatin (Bristol-Myers Squibb Gr. Pharma)

Pms-nystatin (Pharmascience)

Nadostine (Nadeau Laboratories)

Nystatin, 100000 UI/ml / Liquid

Nystatin, 100000 UI/ml / sugar free Liquid

Nystatin, 500000 UI / Tablet

Nystatin, 500000 UI / Tablet

Ofloxacin, $200 \mathrm{mg} /$ Tablet

Ofloxacin, $300 \mathrm{mg} /$ Tablet

Ofloxacin, $400 \mathrm{mg} /$ Tablet

Nadostine (Nadeau Laboratories)

Nadostine (Nadeau Laboratories)

Mycostatin (Bristol-Myers Squibb)

0,80

Apo-Oflox (Apotex)

Apo-Oflox (Apotex)

Apo-Oflox (Apotex)

$\begin{array}{ll} & 3,00 \\ 2,41 & \mathrm{Nd} \\ 2,62 & \mathrm{Nd}\end{array}$

Orciprenaline, $2 \mathrm{mg} / \mathrm{ml} /$ Liquid

Orciprenaline, $2 \mathrm{mg} / \mathrm{ml} /$ Liquid

Oxazepam, $10 \mathrm{mg} /$ Tablet

Alti-orciprenalline (Altimed Pharmaceutical Co.)

Apo-orciprenaline (Apotex)

Novoxapam (Novopharm)

Oxazepam, $15 \mathrm{mg} /$ Tablet

Novoxapam (Novopharm)

Oxtriphylline, $10 \mathrm{mg} / \mathrm{ml} /$ Liquid

Oxtriphylline, $100 \mathrm{mg} /$ Tablet

Oxtriphylline, $20 \mathrm{mg} / \mathrm{ml} /$ Liquid

Choledyl (Parke-Davis)

Novo-Triphyl (Novopharm)

Choledyl (Parke-Davis)

$\mathrm{Nd}$

$\mathrm{Nd}$

0,35

0,096

0,15

0,21

Oxtriphylline, $200 \mathrm{mg} /$ Tablet

Oxtriphylline, $300 \mathrm{mg} /$ Tablet

Oxybutinin, $1 \mathrm{mg} / \mathrm{ml} /$ Liquid

Oxybutinin, $1 \mathrm{mg} / \mathrm{ml} /$ Liquid

Oxybutinin, $5 \mathrm{mg} /$ Tablet

Penicilline G, 500000 U.I. / Tablet

Penicillin VK, 25 mg/ml / Liquid

Penicillin VK, $300 \mathrm{mg} /$ Tablet

Penicillin VK, 500000 U.I. / Tablet

Penicillin VK, 60 mg/ml / Liquid

Penicillin VK, $60 \mathrm{mg} / \mathrm{ml}$ / Liquid

Novo-Triphyl (Novopharm)

Novo-Triphyl (Novopharm)

Apo-oxybutinin (Apotex)

Ditropan (Procter \& Gamble)

Novo-Oxybutinin (Novopharm)

$\mathrm{Nd}$

Novo-Pen G-500 (Novopharm)

0,588

0,566

$\mathrm{Nd}$

Apo-pen VK (Apotex)

Apo-Pen VK (Apotex)

Novo-Pen VK (Novopharm)

Apo-pen VK (Apotex)

Novo-Pen VK (Novopharm)

Neuleptil (Rhône-Poulenc Rorer)

Pericyazin, $10 \mathrm{mg} / \mathrm{ml} /$ Liquid

Phenobarbital, $100 \mathrm{mg}$ / Capsule

Phenobarbital, $15 \mathrm{mg} /$ Tablet

Phenobarbital, $30 \mathrm{mg} /$ Tablet

Phenobarbital, $5 \mathrm{mg} / \mathrm{ml} /$ Liquid

Phenobarbital, $60 \mathrm{mg} /$ Tablet

Phenylbutazone, $100 \mathrm{mg} /$ Tablet

Phenylephrine $\mathrm{HCl}$ - hydrocodone bitartrate - guaifenesin, $4 \mathrm{mg}-1 \mathrm{mg}-40 \mathrm{mg} / \mathrm{ml} /$ Liquid

Novo-Pentobarb (Novopharm)

Phenobarbital (Parke-Davis)

Phenobarbital (Parke-Davis)

Pnenobarbital elixir USP(Stanley Pharmaceuticals)

0,104

2,60

Phenobarbital (Parke-Davis)

Novo-Butazone (Novopharm)

$\mathrm{Nd}$

0,134

0,136

2,81

1,01

0,601

0,104

$\mathrm{Nd}$

$\mathrm{Nd}$

0,501

$\mathrm{Nd}$

2,028

$\mathrm{Nd}$

0,227

0,24

0,33

0,135

$\mathrm{Nd}$

$\mathrm{Nd}$

0,80

$\mathrm{Nd}$

$\mathrm{Nd}$

0,40

0,172

2,27

0,037

0,086

$\mathrm{Nd}$

$\mathrm{Nd}$

$\mathrm{Nd}$

$\mathrm{Nd}$

2,60

0,42

0,43

$\mathrm{Nd}$

$\mathrm{Nd}$

$\mathrm{Nd}$

$\mathrm{Nd}$

0,036

$\mathrm{Nd}$

0,613

0,584

3,66

0,170

2,80

0,220

0,224

$\mathrm{Nd}$

$\mathrm{Nd}$

0,616

0,171

0,81

Novahistex DH Expt (Hoechst Marion Roussel)

0,35

0,053

0,09

0,573

2,50

2,112

1,00

0,254

0,24

0,33

$\mathrm{Nd}$

0,35

0,060

Phenylephrine $\mathrm{HCl}$ - hydrocodone bitartrate,

Novahistine DH (Hoechst Marion Roussel)

3,00

$\mathrm{Nd}$

1,38

$\mathrm{Nd}$ 
Phenylephrine $\mathrm{HCl}$ - hydrocodone bitartrate,

$4 \mathrm{mg}-1 \mathrm{mg} / \mathrm{ml} /$ Liquid

Phenylephrine $\mathrm{HCl}$ - codeine phosphate, $4 \mathrm{mg}-3 \mathrm{mg} / \mathrm{ml} /$ Liquid

Phenytoin sodium, $100 \mathrm{mg} /$ Capsule

Phenytoin sodium, $30 \mathrm{mg} /$ Capsule

Phenytoin, $25 \mathrm{mg} / \mathrm{ml} /$ Liquid

Phenytoin, $50 \mathrm{mg} /$ Tablet

Pindolol, $10 \mathrm{mg} /$ Tablet

Pindolol, $15 \mathrm{mg} /$ Tablet

Pindolol, $5 \mathrm{mg} /$ Tablet

Piroxicam, $10 \mathrm{mg} /$ Capsule

Piroxicam, $20 \mathrm{mg} /$ Capsule

Pivampicillin, $35 \mathrm{mg} / \mathrm{ml} /$ Liquid

Pivampicillin, $500 \mathrm{mg} /$ Tablet

Pivmecillinam, chlorhydrate, $200 \mathrm{mg} /$ Tablet

Polyethylene glycol /electrolytes, / Liquid

Polyethylen glycol /electrolytes, / Liquid

Polyethylen glycol /electrolytes, / Liquid

Polyethylen glycol /electrolytes, / Powder

Polystyrene sulfonate / Liquid

Prazocin HCl, 2 mg / Tablet

Prazocin $\mathrm{HCl}, 5 \mathrm{mg} /$ Tablet

Prednisolone, $1 \mathrm{mg} / \mathrm{ml} /$ Liquid

Prednisolone, $5 \mathrm{mg} /$ Tablet

Prednisone, $5 \mathrm{mg} /$ Tablet

Prednisone, $50 \mathrm{mg} /$ Tablet

Primidone, $125 \mathrm{mg} /$ Chewtab

Primidone, $125 \mathrm{mg} /$ Tablet

Primidone, $250 \mathrm{mg} /$ Comrime

Primidone, $250 \mathrm{mg} /$ Tablet

Prochlorperazine, $1 \mathrm{mg} / \mathrm{ml} /$ Liquid

Prochlorperazine, $10 \mathrm{mg} /$ Tablet

Prochlorperazine, $5 \mathrm{mg} /$ Tablet

Procyclidine, $0,5 \mathrm{mg} / \mathrm{ml} /$ Liquid

Procyclidine, $0,5 \mathrm{mg} / \mathrm{ml} /$ Liquid

Procyclidine, $5 \mathrm{mg} /$ Tablet

Promethazine, $2 \mathrm{mg} / \mathrm{ml} /$ Liquid

Propoxyphene, hydrochloride, $65 \mathrm{mg}$ / Capsule

Propranolol, $10 \mathrm{mg} /$ Tablet

Propranolol, $120 \mathrm{mg} /$ Tablet

Propranolol, $20 \mathrm{mg}$ / Tablet

Propranolol, $40 \mathrm{mg} /$ Tablet

Propranolol, $80 \mathrm{mg} /$ Tablet

Prpoxyphen HCl-ASA-caffeine, / Capsule

Pseudoephedrine, $6 \mathrm{mg} / \mathrm{ml} /$ Liquid

Pseudoephedrine, $6 \mathrm{mg} / \mathrm{ml} /$ Liquid

Psyllium no flavor, / Powder

Psyllium no sugar, / Powder

Psyllium orange flavor / Powder

Psyllium orange, smooth texture / Powder

Psyllium (hydrophilic mucilloid for oral suspension) orange - smooth texture "Sugar free", / Powder

Psyllium no flavor, / Powder
Novahistex DH (Hoechst Marion Roussel)

2,42

$\mathrm{Nd}$

Novahistex C (Hoechst Marion Roussel)

Dilantin (Parke-Davis)

Dilantin (Parke-Davis)

Dilantin-125 (Parke-Davis)

Dilantin Infatabs (Parke-Davis)

Novo-Pindol (Novopharm)

Novo-Pindol (Novopharm)

Novo-Pindol (Novopharm)

Novo-Pirocam (Novopharm)

Novo-Pirocam (Novopharm)

Pondocillin (Leo Pharma)

Pondocillin (Leo Pharma)

Selaxid (Leo Pharma)

Lyteprep (Therapex)

Colyte (Reed \& Carnrick)

PegLyte (Pharmascience)

PegLyte (Pharmascience)

Novo-Prazin (Novopharm)

Novo-Prazin (Novopharm)

Novo-Prazin (Novopharm)

Pediapred (Rhône-Poulenc Rorer)

Novo-Prednisolone (Novopharm)

Novo-Prednisone (Novopharm)

Novo-Prednisone (Novopharm)

Mysoline (Wyeth-Ayerst)

Apo-Primidone (Apotex)

Mysoline(Wyeth-Ayerst)

Apo-Primidone (Apotex)

Stemetil (Rhône-Poulenc Rorer)

Stemetil (Rhône-Poulenc Rorer)

Stemetil (Rhône-Poulenc Rorer)

Pms-procyclidine (Pharmascience)

Kemadrin (Glaxo Wellcome)

Kemadrin (Glaxo Wellcome)

Pms-promethazine (Pharmascience)

Novo-Propoxyn (Novopharm)

Novo-Pranol (Novopharm)

Novo-Pranol (Novopharm)

Novo-Pranol (Novopharm)

Novo-Pranol (Novopharm)

Novo-Pranol (Novopharm)

Novo-Propoxyn Compound (Novopharm)

Balminil Decongestant (Rougier)

Pms-pseudoephedrine (Pharmascience)

Novo-Mucilax (Novopharm)

Novo-Mucilax (Novopharm)

Novo-Mucilax (Novopharm)

Metamucil (Procter \& Gamble)

Metamucil (Procter \& Gamble)

Metamucil (Procter \& Gamble)
$1,98 / \mathrm{g}$

$3,45 / g$

$1,98 / \mathrm{g}$

$\mathrm{Nd} \quad 0,70$

$1,14 \quad \mathrm{Nd}$

$1,92 \quad \mathrm{Nd}$

$0,055 \quad 0,063$

$0,083 \quad 0,094$

$0,050 \quad 0,056$

$1,056 \quad 1,086$

$1,62 \quad 1,62$

$0,43 \quad 0,43$

$0,00 \quad \mathrm{Nd}$

$0,00 \quad \mathrm{Nd}$

$0,00 \quad \mathrm{Nd}$

$0,00 \quad \mathrm{Nd}$

$0,00 \quad \mathrm{Nd}$

$0,202 \quad 0,208$

$0,253 \quad 0,260$

$0,471 \quad 0,484$

2,26 Nd

$0,262 \quad 0,273$

$0,510 \quad 0,520$

$0,586 \quad 0,606$

$\mathrm{Nd} \quad 0,12$

$\mathrm{Nd} \quad 0,17$

$\mathrm{Nd} \quad 0,23$

$\mathrm{Nd} \quad 0,34$

$3,18 \quad \mathrm{Nd}$

$0 \quad \mathrm{Nd}$

$0 \quad \mathrm{Nd}$

$1,16 \quad \mathrm{Nd}$

$0,47 \quad \mathrm{Nd}$

$1,8 \quad \mathrm{Nd}$

$0,491 \quad 0,504$

$0,434 \quad 0,451$

$1,196 \quad 1,240$

$0,352 \quad 0,374$

$0,704 \quad 0,725$

$0,819 \quad 0,861$

$0,371 \quad 0,396$

$\mathrm{Nd} \quad 1,88$

$3,70 \quad \mathrm{Nd}$

$1,983 / \mathrm{g} \quad 1,983 / \mathrm{g}$

$0,000 / \mathrm{g} \quad 0,000 / \mathrm{g}$

$1,617 / \mathrm{g} \quad 1,617 / \mathrm{g}$

$7,76 / \mathrm{g} \quad 7,76 / \mathrm{g}$

$\begin{array}{ll}3,45 / \mathrm{g} & 3,45 / \mathrm{g} \\ 1,98 / \mathrm{g} & 1,98 / \mathrm{g}\end{array}$

$\begin{array}{ll}2,22 & \mathrm{Nd}\end{array}$

$1,056 \quad 1,086$

2,30 Nd


LE JOURNAL CANADIEN DES SCIENCES NEUROLOGIQUES

Drug, concentration and presentation

Commercial name and company

Caloric content* Caloric content*

- CH§ (Kcal)

- Total (Kcal)

Psyllium no flavor, no sugar, smooth texture, / Powder

Metamucil (Procter \& Gamble)

Psyllium + sene, / Granules

Psyllium / Granules

Pyrantel pamoate, $50 \mathrm{mg} / \mathrm{ml} /$ Liquid

Pyridoxine hydrochloride, $25 \mathrm{mg} /$ Tablet

Pyrvinium pamoate, $10 \mathrm{mg} / \mathrm{ml} /$ Liquid

Quinidine, $200 \mathrm{mg} /$ Tablet

Quinine, 200 mg / Capsule

Ranitidine, 15 mg/ml / Liquid

Ranitidine, 150 mg / Tablet

Ranitidine, $150 \mathrm{mg} /$ Tablet

Ranitidine, 300 mg / Tablet

Ranitidine, $300 \mathrm{mg} /$ Tablet

Reserpine, 0,25 mg / Tablet

Rifampin, 150 mg / Capsule

Rifampin, $300 \mathrm{mg} /$ Capsule

Salbutamol, 0,4 mg/ml / Liquid

Salbutamol, $2 \mathrm{mg} /$ Tablet

Salbutamol, $4 \mathrm{mg} /$ Tablet

Secobarbital sodium, $100 \mathrm{mg} /$ Capsule

Selegiline, $5 \mathrm{mg} /$ Tablet

Sennosides, $1,7 \mathrm{mg} / \mathrm{ml} /$ Liquid

Sennosides, $15 \mathrm{mg} / 5 \mathrm{ml}$ (3g) / Granules

Sennosides, 8,6 mg / Tablet

Sennosides, $119 \mathrm{mg} /$ dose unit (70ml) / Liquid

Prodiem Plus (Novartis)

Prodiem Simple (Novartis)

Combantrin (Pfizer)

Vitamine B6 (Wampole)

Vanquin (Warner-Lambert)

Novo-Quinidine (Novopharm)

Novo-Quinine (Novopharm)

Zantac (Glaxo Wellcome)

Novo-Ranidine (Novopharm)

Zantac (Glaxo Wellcome)

Novo-Ranidine (Novopharm)

Zantac (Glaxo Wellcome)

$3,45 / g$

$3,45 / g$

Novo-Reserpine (Novopharm)

Rifadin (Hoechst Marion Roussel)

Rifadin (Hoechst Marion Roussel)

Ventolin (Glaxo Wellcome)

Novo-Salmol (Novopharm)

Novo-Salmol (Novopharm)

$\mathrm{Nd}$

$\mathrm{Nd}$

3,24

0,00

$\mathrm{Nd}$

0,127

0,428

0,80

0,00

0,01

0,00

0,01

Novo-Secobarb (Novopharm)

0,607

0,091

0,181

0,03

0,415

Novo-Selegiline (Novopharm)

Senokot (Purdue Frederick)

Senokot (Purdue Frederick)

Senokot (Purdue Frederick)

X-Prep (Purdue Frederick)

0,829

0,385

0,487

$\mathrm{Nd}$

$\mathrm{Nd}$

$\mathrm{Nd}$

Glysennid (Novartis)

Sennosides, $12 \mathrm{mg} /$ Dragee

Pms-Sennosides (Pharmascience)

Ex-Lax, chocolate pieces (Novartis)

Ex-Lax, sugar coated tablets (Novartis)

X-Prep (Purdue Frederick)

Ex-Lax extra-strong, sugar coated tablets (Novartis)

Glysennid (Novartis)

Pms-Sennosides (Pharmascience)

Ovol (Carter Horner)

Pms-dicitrate sodium (Pharmascience)

Sodium citrate + citric acid, $1 \mathrm{meq} / \mathrm{ml} /$ Liquid

Sodium phosphates, 2,4g monobasic + 0,9g dibasic /5ml / Liquid Fleet Phospho-Soda (Johnson \& Jonhson Merck)

Sodium phosphates, 2,4g monobasic + 0,9g dibasic $/ 5 \mathrm{ml} /$ Liquid Pms-phosphate solution (Pharmascience)

Sotalol HCl, $80 \mathrm{mg} /$ Tablet

Novo-Stalol (Novopharm)

Spiramycine, $250 \mathrm{mg} /$ Capsule

Spiramycine, $500 \mathrm{mg} /$ Capsule

Spironolactone / hydrochlorothiazide, 25/25 mg / Tablet

Spironolactone / hydrochlorothiazide, 50/50 mg / Tablet

Spironolactone, $100 \mathrm{mg} /$ Tablet

Spironolactone, $25 \mathrm{mg} /$ Tablet

Stavudine, $1 \mathrm{mg} / \mathrm{ml} /$ Liquid

Stavudine, $15 \mathrm{mg} /$ Capsule

Stavudine, $20 \mathrm{mg} /$ Capsule

Stavudine, $30 \mathrm{mg} /$ Capsule

Stavudine, $40 \mathrm{mg} /$ Capsule

Sucralfate, $1000 \mathrm{mg} /$ Tablet

Sucralfate, $1000 \mathrm{mg} /$ Tablet

Sucralfate, $200 \mathrm{mg} / \mathrm{ml} /$ Liquid

Rovamycine (Rhône-Poulenc Rorer)

Rovamycine (Rhône-Poulenc Rorer)

Novo-Spirozine (Novopharm)

Novo-Spirozine (Novopharm)

Novo-Spiroton (Novopharm)

Novo-Spiroton (Novopharm)

Zerit (Bristol-Myers Squibb)

Zerit (Bristol-Myers Squibb)

Zerit (Bristol-Myers Squibb)

Zerit (Bristol-Myers Squibb)

Zerit (Bristol-Myers Squibb)

Sulcrate (Hoechst Marion Roussel)

$\mathrm{Nd}$

Novo-Sucralate (Novopharm)

Sulcrate Suspension Plus (Hoechst Marion Roussel)

Dagenan (Rhône-Poulenc Rorer)

\section{$\mathrm{Nd}$}

0,04

1,80

0,74

$\mathrm{Nd}$

0,74

$\mathrm{Nd}$

0,06

$\mathrm{Nd}$

0,05

0,2

0,241

0,92

1,84

1,154

2,308

2,042

0,895

0,195

0,514

0,772

0,772

1,02

0,00

0,144

$0,74 / \mathrm{g}$

$0,72 / \mathrm{g}$

$\mathrm{Nd}$

$\mathrm{Nd}$

0,90

0,228

0,536

0,83

0,138

$\mathrm{Nd}$

0,276

$\mathrm{Nd}$

0,613

$\mathrm{Nd}$

$\mathrm{Nd}$

0,03

0,430

Sulfapyridine, $500 \mathrm{mg} /$ Tablet

2,053

0,860

0,424

0,514

3,04

$2,93 / \mathrm{g}$

0,22

$212 / 70 \mathrm{ml}$

bottle

0,36

$\mathrm{Nd}$

$\mathrm{Nd}$

$\mathrm{Nd}$

38/sachet

$\mathrm{Nd}$

0,34

$\mathrm{Nd}$

0,30

$\mathrm{Nd}$

Nd

$\mathrm{Nd}$

0,253

$\mathrm{Nd}$

$\mathrm{Nd}$

1,188

2,377

2,126

0,929

$\mathrm{Nd}$

$\mathrm{Nd}$

Nd

$\mathrm{Nd}$

$\mathrm{Nd}$

$\mathrm{Nd}$

0,247

$\mathrm{Nd}$

$\mathrm{Nd}$

\begin{tabular}{l} 
\\
\hline \\
\hline 7 \\
\hline 7 \\
\hline 7 \\
\hline
\end{tabular}


Sulfinpyrazone, $100 \mathrm{mg}$ / Tablet

Sulfinpyrazone, $200 \mathrm{mg} /$ Tablet

Sulfisoxazole, $500 \mathrm{mg} /$ Tablet

Sulindac, $150 \mathrm{mg} /$ Tablet

Sulindac, $200 \mathrm{mg} /$ Tablet

Temazepam, $15 \mathrm{mg} /$ Capsule

Tenoxicam, $20 \mathrm{mg} /$ Tablet

Terfenadine, $120 \mathrm{mg} /$ Tablet

Terfenadine, $6 \mathrm{mg} / \mathrm{ml} /$ Liquid

Terfenadine, $60 \mathrm{mg}$ / Tablet

Terfenadine, $60 \mathrm{mg} /$ Tablet

Tetracycline, $25 \mathrm{mg} / \mathrm{ml} /$ Liquid

Tetracycline, $250 \mathrm{mg}$ / Capsule

Tetracycline, $250 \mathrm{mg} /$ Tablet

Tetracycline, chlorhydrate, $250 \mathrm{mg}$ / Capsule

Tetracycline, chlorhydrate, $250 \mathrm{mg} /$ Capsule

Theophylline, 5,33 mg/ml / Liquid

Theophylline, $5,33 \mathrm{mg} / \mathrm{ml} /$ Liquid

Theophylline anhydrous, $100 \mathrm{mg} /$ Tablet

Thioridazine, $10 \mathrm{mg} /$ Tablet

Thioridazine, $100 \mathrm{mg} /$ Tablet

Thioridazine, $2 \mathrm{mg} / \mathrm{ml}$ / Liquid

Thioridazine, $200 \mathrm{mg} /$ Tablet

Thioridazine, $25 \mathrm{mg} /$ Tablet

Thioridazine, $30 \mathrm{mg} / \mathrm{ml} /$ Liquid

Thioridazine, $50 \mathrm{mg} /$ Tablet

Tiaprofenic, acid, $200 \mathrm{mg} /$ Tablet

Tiaprofenic, acid, $300 \mathrm{mg} /$ Tablet

Timolol, maleate, $10 \mathrm{mg} /$ Tablet

Timolol, maleate, $20 \mathrm{mg} /$ Tablet

Timolol, maleate, $5 \mathrm{mg}$ / Tablet

Tolbutamide, $500 \mathrm{mg} /$ Tablet

Tolbutamide, $500 \mathrm{mg} /$ Tablet

Tolmetine sodic, $400 \mathrm{mg} /$ Capsule

Topiramate, $100 \mathrm{mg} /$ Tablet

Topiramate, $200 \mathrm{mg} /$ Tablet

Topiramate, $25 \mathrm{mg} /$ Tablet

Trazadone, $100 \mathrm{mg} /$ Tablet

Trazadone, $150 \mathrm{mg} /$ Tablet

Trazadone, $50 \mathrm{mg} /$ Tablet

Triamterene / Hydrochlorothiazide, 50/25 mg / Tablet

Triazolam, 0,125 mg / Tablet

Triazolam, 0,25 mg / Tablet

Trifluoperazine, $1 \mathrm{mg} /$ Tablet

Trifluoperazine, $10 \mathrm{mg} /$ Tablet

Trifluoperazine, $10 \mathrm{mg} / \mathrm{ml} /$ Liquid

Trifluoperazine, $2 \mathrm{mg}$ / Tablet

Trifluoperazine, $20 \mathrm{mg} /$ Tablet

Trifluoperazine, $5 \mathrm{mg} /$ Tablet

Trihexyphenidyle, $0,4 \mathrm{mg} / \mathrm{ml} /$ Liquid

Trihexyphenidyle, $2 \mathrm{mg} /$ Tablet

Trihexyphenidyle, $5 \mathrm{mg} /$ Tablet

Trimeprazin, 0,5 mg/ml / Liquid

\begin{tabular}{|c|c|c|}
\hline Novo-Pyrazone (Novopharm) & 0,060 & 0,086 \\
\hline Novo-Pyrazone (Novopharm) & 0,603 & 0,629 \\
\hline Novo-Soxazole (Novopharm) & 0,518 & 0,616 \\
\hline Pms-sodium polystyren sulfonate (Pharmascience) & 0,94 & $\mathrm{Nd}$ \\
\hline Novo-Sundac (Novopharm) & 0,055 & 0,094 \\
\hline Novo-Sundac (Novopharm) & 0,102 & 0,126 \\
\hline Novo-Temazepam (Novopharm) & 0,781 & 0,795 \\
\hline Novo-Tenoxicam (Novopharm) & 0,635 & 0,645 \\
\hline Seldane (Hoechst Marion Roussel) & 1,732 & $\mathrm{Nd}$ \\
\hline Seldane (Hoechst Marion Roussel) & 1,44 & $\mathrm{Nd}$ \\
\hline Seldane (Hoechst Marion Roussel) & 0,198 & $\mathrm{Nd}$ \\
\hline Novo-Terfenadine (Novopharm) & 1,453 & 1,483 \\
\hline Novo-Tetra (Novopharm) & 1,98 & 1,98 \\
\hline Novo-Tetra (Novopharm) & 0,163 & 0,217 \\
\hline Novo-Tetra (Novopharm) & 0,064 & 0,110 \\
\hline Apo-Tetra (Apotex) & $\mathrm{Nd}$ & 0,21 \\
\hline Nu-Tetra (Nu-Pharm) & $\mathrm{Nd}$ & 0,21 \\
\hline Theophylline, elixir (Rougier) & $\mathrm{Nd}$ & 2,82 \\
\hline Theolair (3M Pharmaceutical Products) & 2,38 & $\mathrm{Nd}$ \\
\hline Novo-Theophyl SR (Novopharm) & 0,00 & 0,003 \\
\hline Novo-Ridazine (Novopharm) & 0,194 & 0,204 \\
\hline Novo-Ridazine (Novopharm) & 0,083 & 0,117 \\
\hline Mellaril suspension (Novartis pharma) & 2,27 & $\mathrm{Nd}$ \\
\hline Novo-Ridazine (Novopharm) & 0,080 & 0,106 \\
\hline Novo-Ridazine (Novopharm) & 0,082 & 0,093 \\
\hline Mellaril solution (Novartis pharma) & 1,23 & $\mathrm{Nd}$ \\
\hline Novo-Ridazine (Novopharm) & 0,103 & 0,133 \\
\hline Novo-Tiaprofenic (Novopharm) & 0,039 & 0,061 \\
\hline Novo-Tiaprofenic (Novopharm) & 0,059 & 0,092 \\
\hline Novo-Timol (Novopharm) & 0,213 & 0,228 \\
\hline Novo-Timol (Novopharm) & 0,427 & 0,456 \\
\hline Novo-Timol (Novopharm) & 0,107 & 0,114 \\
\hline Novo-Butamide SP.C. (Novopharm) & 0,527 & 0,615 \\
\hline Novo-Butamide (Novopharm) & 0,532 & 0,613 \\
\hline Novo-Tolmetin (Novopharm) & 0,303 & 0,329 \\
\hline Topamax (Jansen-Ortho) & 0,642 & $\mathrm{Nd}$ \\
\hline Topamax (Jansen-Ortho) & 0,350 & $\mathrm{Nd}$ \\
\hline Topamax (Jansen-Ortho) & 0,161 & $\mathrm{Nd}$ \\
\hline Novo-Trazadone (Novopharm) & 0,604 & 0,638 \\
\hline Novo-Trazadone (Novopharm) & 0,147 & 0,163 \\
\hline Novo-Trazadone (Novopharm) & 0,301 & 0,318 \\
\hline Novo-Triamzide (Novopharm) & 0,060 & 0,091 \\
\hline Novo-Triolam (Novopharm) & 0,312 & 0,320 \\
\hline Novo-Triolam (Novopharm) & 0,310 & 0,319 \\
\hline Novo-Flurazine (Novopharm) & 0,267 & 0,278 \\
\hline Novo-Flurazine (Novopharm) & 0,520 & 0,542 \\
\hline Pms-trifluoperazine (Pharmascience) & 2,6 & $\mathrm{Nd}$ \\
\hline Novo-Flurazine (Novopharm) & 0,387 & 0,403 \\
\hline Novo-Flurazine (Novopharm) & 0,523 & 0,545 \\
\hline Novo-Flurazine (Novopharm) & 0,430 & 0,447 \\
\hline Pms-trihexyphenidyle (Pharmascience) & 2,02 & $\mathrm{Nd}$ \\
\hline Novo-Hexidyl (Novopharm) & 0,056 & 0,076 \\
\hline Novo-Hexidyl (Novopharm) & 0,115 & 0,155 \\
\hline Panectyl 2.5 (Rhône-Poulenc Rorer) & 3,22 & $\mathrm{Nd}$ \\
\hline
\end{tabular}


LE JOURNAL CANADIEN DES SCIENCES NEUROLOGIQUES

Trimethoprim + sulfamethoxazole, 160/800 mg / Tablet Trimethoprim + sulfamethoxazole, $160 / 800 \mathrm{mg} /$ Tablet Trimethoprim + sulfamethoxazole, 160/800 mg / Tablet Trimethoprim + sulfamethoxazole, 160/800 mg / Tablet Trimethoprim + sulfamethoxazole, $160 / 800 \mathrm{mg} /$ Tablet Trimethoprim + sulfamethoxazole, $20 / 100 \mathrm{mg} /$ Tablet ped.

Trimethoprim + sulfamethoxazole, $8 / 40 \mathrm{mg} / \mathrm{ml} /$ Liquid

Trimethoprim + sulfamethoxazole, $8 / 40 \mathrm{mg} / \mathrm{ml} /$ Liquid

Trimethoprim + sulfamethoxazole, $8 / 40 \mathrm{mg} / \mathrm{ml} /$ Liquid

Trimethoprim + sulfamethoxazole, $8 / 40 \mathrm{mg} / \mathrm{ml} /$ Liquid

Trimethoprim + sulfamethoxazole, $8 / 40 \mathrm{mg} / \mathrm{ml} /$ Liquid

Trimethoprim + sulfamethoxazole, $80 / 400 \mathrm{mg} /$ Tablet

Trimethoprim + sulfamethoxazole, $80 / 400 \mathrm{mg} /$ Tablet

Trimethoprim + sulfamethoxazole, $80 / 400 \mathrm{mg} /$ Tablet

Trimethoprim + sulfamethoxazole, $80 / 400 \mathrm{mg} /$ Tablet

Trimethoprim + sulfamethoxazole, $80 / 400 \mathrm{mg} /$ Tablet

Trimethoprim, $100 \mathrm{mg}$ / Tablet

Trimethoprim, $200 \mathrm{mg} /$ Tablet

Trimipramine, maleate, $100 \mathrm{mg} /$ Tablet

Trimipramine, maleate, $25 \mathrm{mg} /$ Tablet

Trimipramine, maleate, $50 \mathrm{mg} /$ Tablet

Trovafloxacin, $100 \mathrm{mg} /$ Tablet

Trovafloxacin, $200 \mathrm{mg} /$ Tablet

Valproic acid, $250 \mathrm{mg} /$ Capsule

Valproic acid, $250 \mathrm{mg} /$ Capsule

Valproic acid, $250 \mathrm{mg} /$ Capsule

Valproic acid, $50 \mathrm{mg} / \mathrm{ml} /$ Liquid

Valproic acid, $50 \mathrm{mg} / \mathrm{ml} /$ Liquid

Valproic acid, $500 \mathrm{mg} /$ Capsule

Valproic acid, $500 \mathrm{mg} /$ Capsule

Valproic acid, $500 \mathrm{mg} /$ Capsule

Valproic acid, $500 \mathrm{mg} /$ Capsule

Valproic acid, $50 \mathrm{mg} / \mathrm{ml} /$ Liquid

Verapamil, $120 \mathrm{mg} /$ Tablet

Verapamil, $80 \mathrm{mg} /$ Tablet

Vigabatrin, $500 \mathrm{mg} /$ Tablet

Vitamine a b c d, / Liquid

Vitamine a b c d, / Liquid

Vitamine a b c d, / Liquid

Vitamine a c d, / Liquid

Vitamine a c d + fluor, / Liquid

Vitamine a c d + fluor, / Liquid

Vitamine d, $400 \mathrm{u} / \mathrm{ml} /$ Liquid

Vitamine e, $50 \mathrm{u} / \mathrm{ml} /$ Liquid

Vitamine e, 100 UI / Capsule

Vitamine e, 400 UI / Capsule

Vitamines, multi-, / Capsule

Vitamines, multi-, / Tablet

Vitamines, multi-, / Tablet

Zidovudine, 100 mg / Capsule

\begin{tabular}{|c|c|c|}
\hline Apo-sulfatrim DS (Apotex) & $\mathrm{Nd}$ & 0,30 \\
\hline Bactrim Roche (Hoffman-La Roche) & $\mathrm{Nd}$ & 0,40 \\
\hline Nu-Cotrimox (Nu-Pharm) & $\mathrm{Nd}$ & 0,30 \\
\hline Septra DS (Glaxo Wellcome) & $\mathrm{Nd}$ & 0,08 \\
\hline Novo-Trimel (Novopharm) & 0,325 & 0,470 \\
\hline Apo-sulfatrim (Apotex) & $\mathrm{Nd}$ & 0,04 \\
\hline Apo-sulfatrim (Apotex) & $\mathrm{Nd}$ & 2,47 \\
\hline Nu-Cotrimox (Nu-Pharm) & $\mathrm{Nd}$ & 2,47 \\
\hline Septra (Glaxo Wellcome) & $\mathrm{Nd}$ & 2,60 \\
\hline Novo-trimel (Novopharm) & 0,011 & 0,011 \\
\hline Bactrim Roche (Hoffman-La Roche) & 1,81 & 2,42 \\
\hline Apo-sulfatrim (Apotex) & $\mathrm{Nd}$ & 0,15 \\
\hline Bactrim Roche (Hoffman-La Roche) & $\mathrm{Nd}$ & 0,20 \\
\hline Nu-Cotrimox (Nu-Pharm) & $\mathrm{Nd}$ & 0,15 \\
\hline Septra (Glaxo Wellcome) & $\mathrm{Nd}$ & 0,04 \\
\hline Novo-Trimel (Novopharm) & 0,163 & 0,235 \\
\hline Proloprim (Glaxo Wellcome) & 0,52 & $\mathrm{Nd}$ \\
\hline Proloprim (Glaxo Wellcome) & 0,12 & $\mathrm{Nd}$ \\
\hline Novo-Tripramine (Novopharm) & 0,046 & 0,067 \\
\hline Novo-Tripramine (Novopharm) & 0,012 & 0,017 \\
\hline Novo-Tripramine (Novopharm) & 0,023 & 0,033 \\
\hline Trovan (Pfizer) & 0,388 & $\mathrm{Nd}$ \\
\hline Trovan (Pfizer) & 0,780 & $\mathrm{Nd}$ \\
\hline Depakene (Abbott Laboratories) & 0,00 & $\mathrm{Nd}$ \\
\hline Pms-Valproic Acid (Pharmascience) & 0,71 & $\mathrm{Nd}$ \\
\hline Novo-Valproic (Novopharm) & 0,90 & $\mathrm{Nd}$ \\
\hline Alti-Valproic (AltiMed Pharmaceutical Company) & $\mathrm{Nd}$ & 3,54 \\
\hline Pms-Valproic Acid (Pharmascience) & 2,8 & $\mathrm{Nd}$ \\
\hline Depakene (Abbot) & 0,00 & $\mathrm{Nd}$ \\
\hline Pms-Valproic Acid E.C. (Pharmascience) & 0,00 & $\mathrm{Nd}$ \\
\hline Pms-Valproic Acid (Pharmascience) & 0,71 & $\mathrm{Nd}$ \\
\hline Novo-Valproic (Novopharm) & 0,88 & $\mathrm{Nd}$ \\
\hline Depakene (Abbott Laboratories) & 2,97 & 3,54 \\
\hline Novo-Veramil (Novopharm) & 0,083 & 0,096 \\
\hline Novo-Veramil (Novopharm) & 0,055 & 0,064 \\
\hline Sabril (Hoechst Marion Roussel) & 0,056 & 0,579 \\
\hline Infantol drops (Carter Horner) & $\mathrm{Nd}$ & 2,8 \\
\hline Infantol liquid (Carter Horner) & $\mathrm{Nd}$ & 1,16 \\
\hline Polyvisol (Mead Johnson) & 3,40 & $\mathrm{Nd}$ \\
\hline Tri-vi-sol (Mead Johnson) & 2,20 & $\mathrm{Nd}$ \\
\hline Tri-vi-flor (Mead Johnson) & 2,20 & $\mathrm{Nd}$ \\
\hline Tri-vi-sol with fluor (Mead Johnson) & 2,20 & $\mathrm{Nd}$ \\
\hline D-vi-sol 400 u/0.6 ml (Mead Johnson) & 2,20 & $\mathrm{Nd}$ \\
\hline Aquasol e (Novartis) & 1,52 & $\mathrm{Nd}$ \\
\hline Aquasol e (Novartis) & 1,44 & $\mathrm{Nd}$ \\
\hline Vitamine E (Santé Naturelle ${ }^{\mathrm{TM}}$ Adrien Gagnon) & 0,29 & $\mathrm{Nd}$ \\
\hline Fortamines-10 Capsule (Rougier) & 0,36 & $\mathrm{Nd}$ \\
\hline Fortamines-10 (Rougier) & 0,36 & $\mathrm{Nd}$ \\
\hline Maxi-10 (Rougier) & 0,36 & $\mathrm{Nd}$ \\
\hline Novo-AZT (Novopharm) & 0,060 & 0,074 \\
\hline
\end{tabular}

* The caloric content indicated is for one $\mathrm{ml}$, one tablet or one capsule unless otherwise indicated. $\mathrm{CH}^{\S}=$ carbohydrate.

$\mathrm{Nd}=$ No data. When no data is available for caloric content provided by carbohydrates, we suggest the use of total caloric content. 
Table 2: Worst case scenario study data

\begin{tabular}{|c|c|c|c|c|c|c|c|}
\hline Medication* & Formulation & $\begin{array}{l}\text { Active } \\
\text { ingredient (mg) }\end{array}$ & Weight (g) & $\begin{array}{l}\text { Excipient } \\
\text { weight }(\mathrm{g})\end{array}$ & $\begin{array}{l}\text { Caloric } \\
\text { content, } \\
\text { estimated (kCal) }\end{array}$ & $\begin{array}{l}\text { Caloric } \\
\text { content (kCal) }\end{array}$ & $\begin{array}{l}\text { Difference } \\
(\mathrm{kCal})\end{array}$ \\
\hline
\end{tabular}

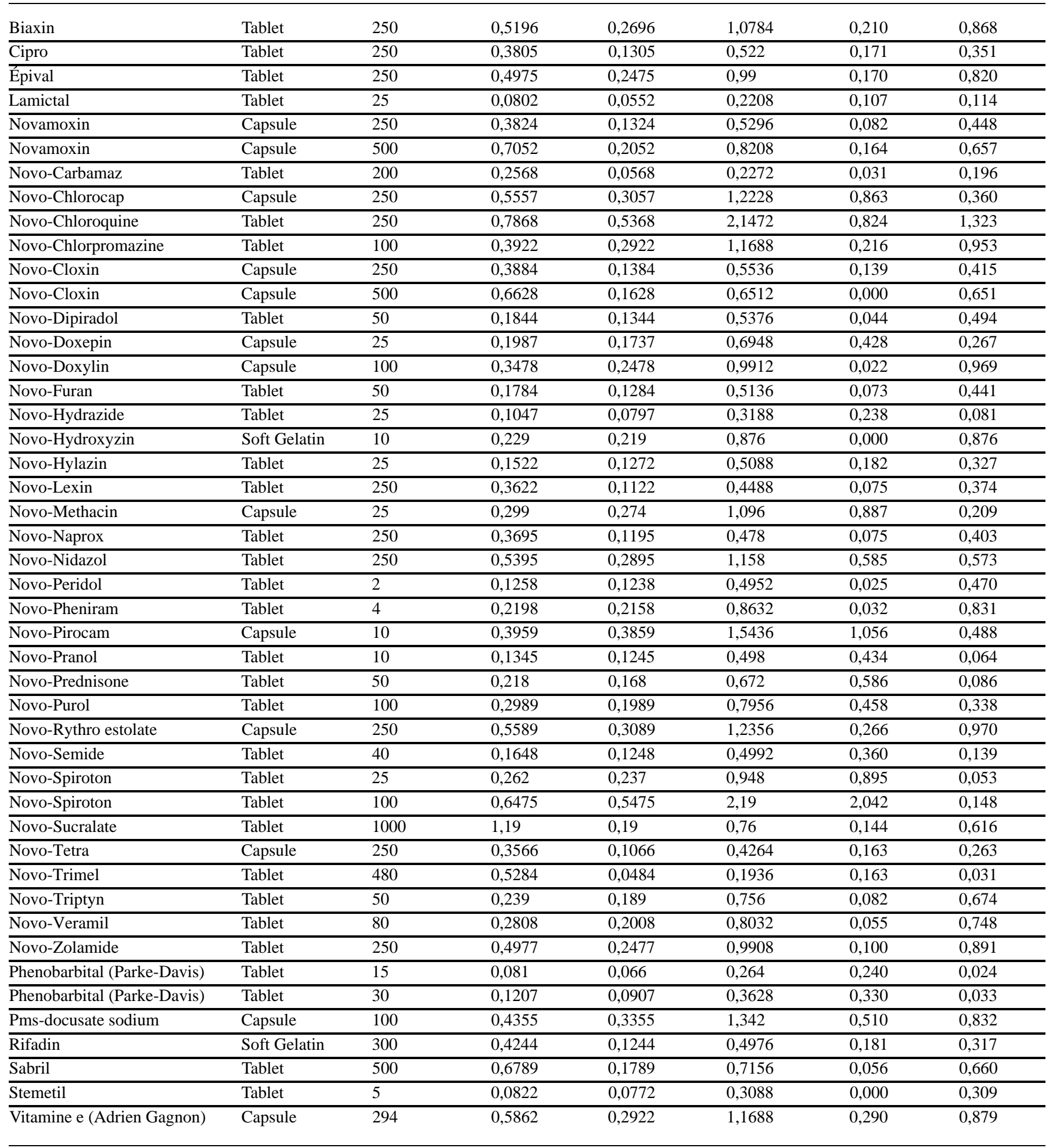

* Trade names. 